\title{
Green synthesized titanium dioxide nanoparticles and their future applications in biomedicine, agriculture and industry
}

\section{Bogdan Andrei Miu, Anca Dinischiotu ${ }^{凶}$}

\author{
Department of Biochemistry and Molecular Biology, Faculty of Biology, University of Bucharest, 91-95 Splaiul \\ Independenței, 050095 Bucharest, Romania
}

\begin{abstract}
${ }^{\circledR}$ Correspondence to: Anca Dinischiotu, Department of Biochemistry and Molecular Biology, Faculty of Biology, University of Bucharest, 91-95 Splaiul Independentei, 050095 Bucharest, Romania E-mail: anca.dinischiotu@bio.unibuc.ro
\end{abstract}

Received: 3 February 2021 / Revised: 26 March 2021 / Accepted: 22 April 2021 / Available online: 21 May 2021

\begin{abstract}
In the green synthesis of titanium dioxide nanoparticles $\left(\mathrm{TiO}_{2} \mathrm{NPs}\right)$ chemical reagents are replaced with biological extracts. Conventional methods used in the manufacture of $\mathrm{TiO}_{2} \mathrm{NPs}$ raise environmental issues as they use harmful chemicals and spend a high amount of energy. At a laboratory scale, biologically synthesized titanium dioxide nanoparticles (bio- $\mathrm{TiO}_{2} \mathrm{NPs}$ ) proved to be a suitable alternative to the chemically synthesized ones. The biological activity of NPs is mainly determined by their shape, size and crystalline structure. However, these characteristics are hardly controlled when natural sources of reagents are used and so bio- $\mathrm{TiO}_{2} \mathrm{NPs}$ did not reach an advanced technology readiness level. In this paper, we reviewed the majority of the available studies referring to bio-TiO $\mathrm{NPs}_{\text {. }}$ Our aim is to briefly present the efficiency of biochemicals from different living organisms in producing $\mathrm{TiO}_{2}$ nanoscale particles as well as the benefits bio- $\mathrm{TiO}_{2} \mathrm{NPs}$ would bring to the biomedical, agricultural and industrial sectors. Finally, based on the available data we discuss the sustainability of bio- $\mathrm{TiO}_{2}$ NPs referring to their possible environmental, economic and societal impacts.
\end{abstract}

Keywords: titanium dioxide, nanoparticles, green synthesis, sustainability

\section{Introduction}

Titanium dioxide nanoparticles $\left(\mathrm{TiO}_{2} \mathrm{NPs}\right)$ are some of the most produced and used nanomaterials worldwide. Products containing $\mathrm{TiO}_{2}$ NPs are already available on the market (Robichaud et al., 2009; Inshakova and Inshakov, 2017). Also, applications involving them in agriculture (Rodríguez-González et al., 2019), medicine (Ziental et al., 2020) and energetical field (Hou et al., 2019) are currently under development. Therefore, the production volume of $\mathrm{TiO}_{2} \mathrm{NPs}$ is predicted to grow in the near future in order to satisfy the increased demand. As the majority of human activities, the industry of NPs has a serious negative impact on the environment. According to estimations made in 2018, the global greenhouse emissions of $\mathrm{TiO}_{2}$ NPs manufacturing process were comparable to that of automobile manufacturing industry in the United States (Wu et al., 2019).

The negative environmental impact of NPs synthesis derives from the conventional techniques used by manufacturers. These usually require high energy consumption and harmful reagents (Jamkhande et al., 2019).
In an attempt to prevent the increase of emissions of NPs manufacturing, researchers explored the potential of natural extracts as reagents instead of chemical ones.

The techniques that involve extracts from microorganisms or different plant parts are important in the framework of the biological/green NPs synthesis methods. Broadly, NPs are formed through the action of biochemicals on metal ions provided by a precursor salt. In the case of $\mathrm{TiO}_{2} \mathrm{NPs}$, the precursor can be represented by titanium tetraisopropoxide (Landage et al., 2020), metatitanic acid (Alavi and Karimi, 2018), titanium oxysulfate (Subhapriya and Gomathipriya, 2018) or bulk forms of $\mathrm{TiO}_{2}$ (He et al., 2017). If we go further, agrowastes or organic matter waste may represent a sustainable source of biomolecules for the green synthesis of NPs (Zamani et al., 2019).

However, biological techniques remained at a laboratory scale mostly because the morphology of NPs is hard to be controlled. The biological activity of $\mathrm{TiO}_{2} \mathrm{NPs}$ is mainly determined by their shape, size and crystalline structure. However, there is evidence that bio- $\mathrm{TiO}_{2} \mathrm{NPs}$ present on their surface biomolecules from the biological extract used (Kaur et al., 2019). So far, it is unknown whether this coating brings new properties to green NPs. 
Even though green synthesis methods are clearly economically feasible and have a low impact on environment, there are concerns regarding their final products, the nano-scale particles. Public acceptance of NPs is mainly impeded by their possible toxic effects. However, experimental data referring to the toxicity of green $\mathrm{TiO}_{2}$ NPs are nearly inexistent. Research focused more on the applications of bio- $\mathrm{TiO}_{2} \mathrm{NPs}$ and in many cases, they proved to be a viable alternative to the chemically synthesized ones.

\section{Conventional methods used for $\mathrm{TiO}_{2}$ NPs synthesis}

As time went on, intense research efforts in nanotechnology have led to the designing of a wide variety of synthesis approaches that are available nowadays for the production of nanoscale particles. IUPAC defines a NP as a particle with dimensions between 1 and $100 \mathrm{~nm}$ (Vert et al., 2012). Thus, there are two different possibilities to build NPs, depending on "the starting point". One cluster of synthesis methods are defined by a top-down approach consisting in the reduction in size of a larger structure represented by the bulk material. The other option is to start from lower size particles such as atoms and molecules and assemble them until the new structure reach the dimension of a NP. The last one represents the principle of the bottom-up approach. Also, NPs synthesis methods can be classified as physical, chemical or biological (green synthesis or bio-assisted synthesis) methods, depending on the nature of treatments and processes involved (Ijaz et al., 2020). Generating NPs by physical methods implies the use of mechanical (Yadav et al., 2015), thermal (Sofronov et al., 2020), radiation (Singh et al., 2016) or electrical energy (Kumar et al., 2019), processes usually making use of the top-down principle. Other common physical methods that led to the formation of metal oxide NPs include ball milling, sputtering, electrospraying, laser ablation and laser pyrolysis. These techniques are different and each one presents advantages and disadvantages. However, there are a few physical methods such as the flame spray pyrolysis that are classified as bottom-up methods. Overall, the major disadvantage of physical methods is that they are less economical. Processes require a huge amount of energy, expensive equipment and an abundant waste is produced. On the other side, these methods can be used to obtain a large range of materials, contamination does not occur and NPs obtained have particular properties depending on the method used (Jamkhande et al., 2019).

The synthesis of NPs through chemical techniques is usually done by bottom-up methods. Examples of the most common chemical methods used to synthesise metallic oxide NPs are sol gel method, microemulsion technique, hydrothermal method, polyol synthesis and chemical vapor synthesis. The major disadvantage of chemical methods is that they use substances which are corrosive, toxic, harmful for human health and also may pollute the environment having concerning consequences. On the other hand, the parameters (morphology, size, shape) of NPs produced by chemical methods are easily controlled and the techniques are relatively simple (Jamkhande et al., 2019).

\section{Green approaches and natural sources of biochemicals used for synthesis of $\mathrm{TiO}_{2}$ NPs}

Nowadays, biological methods are gaining more attention due to their characteristics which make them a viable eco-friendly, simple and cost-effective alternative to chemical and physical methods (Wong-Pinto et al., 2020). Practices involving living organisms in the synthesis of NPs are also known as green synthesis methods because they are in accordance with the principles of green chemistry, a concept of sustainable development addressed to the molecular level.

Ten year ago, the Twelve Principles of green chemistry have been stated, which can be summarised as: 1) waste prevention, 2) atom economy (to maximise the fraction of the used raw materials), 3) less hazardous chemical synthesis, 4) safer chemicals, 5) safer solvents and auxiliaries, 6) energy efficiency, 7) renewable feedstocks, 8) reduced derivatives, 9) use catalytic reagents, 10) design biodegradable materials and chemicals, 11) pollution prevention and 12) accident prevention (Anastas and Eghbali, 2010).

The majority of studies implement a NPs synthesis approach in two steps: 1) preparation of a biological reagent and 2) the synthesis of NPs.

Generally, aqueous plant extracts are preferred, although ethanol is sometimes used as a solvent. To prepare this type of extract, a plant part is collected, washed, dried and grinded. The obtained powder is mixed with a solvent and usually boiled for a couple of minutes. The extract and biological waste material are separated by filtration or centrifugation. On the other hand, microorganisms can be also involved in the green synthesis of NPs as intact cells or a filtrate obtained from a liquid culture.

To synthesise metallic NPs, the biological reagent is mixed with a precursor containing metal ions. The biochemicals from the biological reagent will start converting ions into nuclei in a time dependent manner. A constant deposition of ions on the nuclei will lead to the increase of their dimensions up to the nanoscale (Tehrani et al., 2019). Previously it was observed that properties of the green NPs, such as their morphology, depend on the amount of biochemicals extracted, the ratio between biochemicals reducing agents and quantity of ions, the period of time in which they are in contact, temperature, $\mathrm{pH}$ of the mixture as well as exposure time to light (Shankar et al., 2014; Kumari et al., 2016; Velgosová et al., 2016; Satpathy et al., 2019). 
Mostly, the biological synthesis of NPs is based on the reducing power of microorganisms and plants extracts. As each species is able to synthesize different biomolecules, the reducing power and yield differ from one organism to other.

Broadly, the biological activity of NPs is dependent on their size and shape. For $\mathrm{TiO}_{2} \mathrm{NPs}$ the crystalline phase is also specific. $\mathrm{TiO}_{2}$ crystals can be packed into the form of anatase, rutile or brookite, each with different features.

\section{Bacterial species involved in the synthesis of $\mathrm{TiO}_{2}$ NPS}

Once biological entities have been involved in the nanotechnological field, bacteria proved that can act as important nanofactories. First, bacteria possess an intense ion reducing activity due to their enzymatic system which is able to convert different metal salts into nanoscale metal structures (Kalimuthu et al., 2008; Bai et al., 2009). Secondly, hazardous chemicals are not used in bacterial mediated synthesis and the costs are minimal. Moreover, bacterial cultures are easy to handle and obtain as bacterial cells usually have a high growth rate. Also, the parameters influencing their growth (e.g. temperature, nutrients) are controlled without difficulties in an artificial environment.

Studies performing bacterial mediated synthesis of $\mathrm{TiO}_{2}$ NPs used organisms belonging to different genera such as: Aeromonas (Jayaseelan et al., 2013), Bacillus (Kirthi et al., 2011; Órdenes-Aenishanslins et al., 2014; Khan and Fulekar, 2016; Jalali et al., 2020), Halomonas (Taran et al., 2018), Propionibacterium (Babitha and Korrapati, 2013), Staphylococcus (Landage et al., 2020) and Streptomyces (Ağçeli et al., 2020). So far, several species of the genus Bacillus were involved in the green synthesis of $\mathrm{TiO}_{2}$ NPs and all led to different performances. $\mathrm{TiO}_{2} \mathrm{NPs}$ synthesized in a culture of $B$. thuringiensis had a size of 33-44 nm and Fouriertransform infrared spectroscopy (FTIR) analysis suggested that they are coupled with phenolic, alcoholic, carboxylic groups and aliphatic amines as well as lipids and proteins. The analysis also suggested that water droplets are present on their surface (Jalali et al., 2020). Scanning electronic microscopy (SEM) images revealed $\mathrm{TiO}_{2}$ NPs within the size range of 50 to $90 \mathrm{~nm}$ when $B$. subtilis cells were used. The evaluation showed that the biomolecules on the surface were not so diverse (Kirthi et al., 2011). A similar result was obtained by ÓrdenesAenishanslins et al. (2014) when $\mathrm{TiO}_{2}$ NPs were synthesized using $B$. mycoides. The study of Khan and Fulekar (2016) on $\mathrm{TiO}_{2}$ NPs synthesis using $B$. amyloliquefaciens suggested that the extracellular bacterial enzyme alpha amylase plays a significant role in the formation and stabilization of these NPs. The reaction between $B$. amyloliquefaciens cells and titanyl sulphate resulted in $\mathrm{TiO}_{2}$ NPs with a variable size (22.11-97.28 $\mathrm{nm})$ and a cristaline anatase structure. It is important to mention that, in order to boost the photocatalytic performances of the resulted NPs, they were doped with different metals such as: platinum, lanthanum, zinc and silver (Khan and Fulekar, 2016). The ability of alpha amylase to assist the biosynthesis of $\mathrm{TiO}_{2} \mathrm{NPs}$ was also confirmed by Ahmad et al. (2015). They proposed that the proline residues of this enzyme play a crucial role in the $\mathrm{TiO}_{2} \mathrm{NPs}$ biosynthesis mechanism. More specifically, the titanyl hydroxide or titanyl sulphate (or any other precursor) is protonated due to a hydrogen ion from this amino acid which is picked up by a pair of electrons from the oxygen in hydroxyl group of the precursor salt. Further, a water molecule detaches from the precursor, causing the reduction of titanium to the ionic form $\mathrm{Ti}^{3+}$. The proline chemical structure recovers by picking up the hydrogen ion from the $\mathrm{Ti}$ compound and thus the formation of $\mathrm{TiO}_{2}$ takes place (Ahmad et al., 2015). The same mechanism was proposed also by Jayaseelan et al. (2013) that studied the formation of $\mathrm{TiO}_{2}$ NPs assisted by Aeromonas hydrophila. Gas chromatography-mass spectrometry (GC-MS) analyses revealed that the dipeptide glycyl-L-proline was the major constituent of the liquid culture used. Other found minor constituents (1Leucyl-d-leucine, glycyl-L-glutamic acid and uric acid) also include carboxyl and carbonyl groups which are thought to be involved in the reducing and capping of NPs (Jayaseelan et al., 2013). It is necessary to mention that besides amylase other enzymes such as urease (Johnson et al., 2012) and lysozyme (Luckarift et al., 2006) were found to be involved in NPs biosynthesis process.

As precursor titanium salts could harm bacteria and so affect the success of NPs formation, the use of heavy metal resistant microorganisms could solve this issue. The pressure of hostile environments forced bacteria to adapt by evolving molecular mechanisms aiming to solubilize and accumulate metals (Jose et al., 2011; Lopes et al., 2020). For example, Propionibacterium jensenii strain isolated from coal fly ash effluent and screened for different heavy metal salts was effectively used to synthesise anatase- $\mathrm{TiO}_{2} \mathrm{NPs}$ with a size range between 15 to $80 \mathrm{~nm}$ as SEM images shown (Babitha and Korrapati, 2013). $\mathrm{TiO}_{2}$ NPs were also synthesized using a strain of $B$. mycoides originating from the soil of a volcanic area (Órdenes-Aenishanslins et al., 2014).

Another method developed to obtain $\mathrm{TiO}_{2}$ NPs used a strain of Streptomyces isolated from soil samples. It was noticed that concentration of titanyl hydroxide (used as a precursor) had no significant effect on the size distribution of $\mathrm{TiO}_{2}$ NPs, but changed the yield of reaction. Therefore, a proportion of $2: 1$ between the titanyl hydroxide solution and bacterial culture was found to maximize the production of $\mathrm{TiO}_{2} \mathrm{NPs}$, but just at a $\mathrm{pH}$ of 6.5. Increased $\mathrm{pH}$ values above this limit caused a decline in the production rate of NPs (A ğçeli et al., 2020). The biosynthesis of $\mathrm{TiO}_{2}$ NPs was reported also using Staphylococcus aureus (Landage et al., 2020) and Halomonas elongata (Taran et al., 2018). 
All studies mentioned in this section are summarized in Table 1 together with characteristics of the obtained NPs and their possible applications.

Table 1. Bacterial species involved in $\mathrm{TiO}_{2} \mathrm{NPs}$ synthesis

\begin{tabular}{|c|c|c|c|c|c|}
\hline Bacterial species & $\begin{array}{l}\text { Titanium } \\
\text { precursor }\end{array}$ & $\begin{array}{l}\text { Size (nm) } \\
\text { and shape }\end{array}$ & $\begin{array}{l}\text { Crystalline } \\
\text { phase }\end{array}$ & Application & Reference \\
\hline Aeromonas hydrophila & $\mathrm{TiO}(\mathrm{OH})_{2}$ & $\begin{array}{l}28-54 \\
\text { round }\end{array}$ & rutile & antibacterial activity & Jayaseelan et al. (2013) \\
\hline $\begin{array}{l}\text { Bacillus } \\
\text { amyloliquefaciens }\end{array}$ & $\mathrm{TiOSO}_{4}$ & $\begin{array}{c}\text { 22.11- } \\
97.28 ; \\
\text { spherical }\end{array}$ & anatase & $\begin{array}{l}\text { photocatalytic degradation } \\
\text { of reactive red } 31 \text { dye }\end{array}$ & $\begin{array}{l}\text { Khan and Fulekar } \\
(2016)\end{array}$ \\
\hline Bacillus mycoides & $\mathrm{TiO}(\mathrm{OH})_{2}$ & $\begin{array}{c}40-60 ; \\
\text { spherical }\end{array}$ & anatase & $\begin{array}{c}\text { antibacterial activity; } \\
\text { potential use in solar cells }\end{array}$ & $\begin{array}{l}\text { Órdenes-Aenishanslins } \\
\text { et al. (2014) }\end{array}$ \\
\hline Bacillus subtilis & $\mathrm{TiO}(\mathrm{OH})_{2}$ & $\begin{array}{c}66-77 ; \\
\text { spherical }\end{array}$ & anatase & - & Kirthi et al. (2011) \\
\hline Bacillus thuringiensis & $\mathrm{TiO}(\mathrm{OH})_{2}$ & $\begin{array}{l}33-44 ; \\
\text { spherical }\end{array}$ & $\begin{array}{l}\text { anatase and } \\
\text { rutile }\end{array}$ & $\begin{array}{l}\text { increasing the persistence } \\
\text { of a microbial insecticide }\end{array}$ & Jalali et al. (2020) \\
\hline Halomonas elongate & $\mathrm{TiO}(\mathrm{OH})_{2}$ & $\begin{array}{c}104.63 \\
\pm 27.75 \\
\text { spherical }\end{array}$ & anatase & antibacterial activity & Taran et al. (2018) \\
\hline $\begin{array}{l}\text { Propionibacterium } \\
\text { Jensenii }\end{array}$ & $\mathrm{TiO}(\mathrm{OH})_{2}$ & $\begin{array}{l}15-80 \\
\text { spherical }\end{array}$ & anatase & wound healing potential & $\begin{array}{l}\text { Babitha and Korrapati } \\
\text { (2013) }\end{array}$ \\
\hline Staphylococcus aureus & TTIP & $\begin{array}{c}10-30 \\
\text { spherical }\end{array}$ & anatase & antibacterial activity & Landage et al. (2020) \\
\hline Streptomyces sp. & $\mathrm{TiO}(\mathrm{OH})_{2}$ & $\begin{array}{c}43-67 ; \\
\text { spherical }\end{array}$ & anatase & $\begin{array}{c}\text { antibacterial and } \\
\text { antifungal activity }\end{array}$ & Ağçeli et al. (2020) \\
\hline
\end{tabular}

TTIP- titanium isopropoxide; $\mathrm{TiOSO}_{4}$ - titanium oxysulfate; $\mathrm{TiO}(\mathrm{OH})_{2}$ - metatitanic acid.

\section{Fungi and yeast species involved in the synthesis of $\mathrm{TiO}_{2} \mathrm{NPS}$}

Some unique features that make microfungi suitable for NPs production were revealed so far. First of all, fungal cultures, as bacterial ones, does not require high amounts of financial or energetical resources. They are easily obtained and handled and their use makes the synthesis of NPs greener. Compared to other microorganisms, microfungi can easily grow in bioreactors, the mycelial biomass being increased as well as the yield of NPs production. Fungal mediated synthesis of NPs is mostly based on the amount of extracellularly released enzymes (Alghuthaymi et al., 2015; Guilger-Casagrande et al., 2019). However, the synthesis can be performed also intracellularly, fungal cells showing higher accumulation yields which are based on a high affinity of the cell walls for metal salts. Not least, microfungi tolerate high amounts of metals when compared to bacterial cells (Hulkoti and Taranath, 2014).

In the past years some fungal strains were studied for their ability to reduce titanium oxide to nanoscale particles. So far, species of Aspergillus (Rajakumar et al., 2012a; Raliya et al., 2015), Fomes (Rehman et al., 2020) and Trichoderma (Chinnaperumal et al., 2018) were involved in studies that performed the fungal mediated synthesis of $\mathrm{TiO}_{2}$ NPs. FTIR spectrum revealed that the culture filtrate of $T$. viride contained a lot of possible functional groups that could produce the reduction of titanyl hydroxide, including the $\mathrm{O}-\mathrm{H}$ and $\mathrm{C}=\mathrm{O}$ bonds.
Analysis showed spherical rutile- $\mathrm{TiO}_{2}$ NPs with diameters between 60 and $86.67 \mathrm{~nm}$. No agglomerates were observed and $\mathrm{TiO}_{2} \mathrm{NPs}$ kept scattered revealing that T. viride has a good capping activity (Chinnaperumal et al., 2018). Fomes fomentarius assisted synthesis of $\mathrm{TiO}_{2}$ NPs did not produce favorable results as the NPs were too large, exceeding the limit of $100 \mathrm{~nm}$ and formed aggregates. Interestingly, in the same study $F$. fomentarius was used for the synthesis of silver NPs with a completely different size range (10-20 nm), but they were also agglomerated which indicate an inefficient capping process (Rehman et al., 2020). However, the mechanisms of fungi mediated synthesis of NPs is unclear and there are no other studies to compare the efficiency of $F$. fomentarius in $\mathrm{TiO}_{2} \mathrm{NPs}$ formulation.

Two studies performing the synthesis of $\mathrm{TiO}_{2} \mathrm{NPs}$ through a similar approach involved each a different strain of Aspergillus flavus. However, the characteristics of NPs were dramatically different. Raliya et al. (2015) obtained NPs with a diameter ranging from 12 to $15 \mathrm{~nm}$ while Rajakumar et al. (2012a) observed that NPs have a size between 62 and $74 \mathrm{~nm}$. The difference may be explained by the amount of biomolecules included in the reaction mixture. In general, it was observed that using high concentrations of biochemicals lead to the formation of larger NPs (Kumari et al., 2016; Jain and Mehata, 2017). Whereas bacteria synthesized predominantly $\mathrm{TiO}_{2}$ NPs in anatase form (Kirthi et al., 2011; Taran et al., 2018; Landage et al., 2020) fungi mediated rutile form of these NPs (Chinnaperumal et al., 2018; Rehman et al., 
2020). Other studies revealed that Saccharomyces cerevisiae generated $\mathrm{TiO}_{2}$ NPs in anatase form. Other researchers were able to convert amorphous bio- $\mathrm{TiO}_{2}$ NPs into anatase or rutile by calcination, the crystalline phase obtained being dependent on the temperature applied. For example, Jalali et al. (2020) observed that calcination at $450^{\circ} \mathrm{C}$ led to anatase- $\mathrm{TiO}_{2}$, but increasing the temperature up to $1200^{\circ} \mathrm{C}$ would cause the conversion of anatase into rutile (Jalali et al., 2020). However, Bao et al. (2012) demonstrated that the crystal phase of $\mathrm{TiO}_{2}$ NPs cannot be changed through thermic treatments up to $750^{\circ} \mathrm{C}$, but the biotemplates used could determine the NPs structure. In their experiment, biosynthesis reaction performed using yeast or albumen has led to the formation of anatase- $\mathrm{TiO}_{2} \mathrm{NPs}$ while rutile- $\mathrm{TiO}_{2} \mathrm{NPs}$ were obtained using dandelion pollen, these results being unchanged at different temperatures from $250^{\circ} \mathrm{C}$ to $750^{\circ} \mathrm{C}$ (Bao et al., 2012). The same situation was observed by Peiris et al. (2018) with thermic treatments of $\mathrm{TiO}_{2}$ NPs synthesized by Saccharomyces cerevisiae. In all cases an anatase crystalline phase of $\mathrm{TiO}_{2}$ NPs was obtained (Peiris et al., 2018).

In general, yeast cells can easily cope with high levels of metal toxicity. All yeast genera possess detoxification mechanisms that allow them to survive in such conditions and accumulate intracellularly certain heavy metal ions. These detoxification mechanisms are based on reductive enzymes, chelation with extracellular biomolecules or control of ion flux through the cell wall and membrane (Rana et al., 2020). To the best of our knowledge Saccharomyces cerevisiae is the only yeast species that was involved in the green synthesis of $\mathrm{TiO}_{2}$ NPs. In general, these studies reported the formation of NPs with a small size, up to $20 \mathrm{~nm}$. Peiris et al. (2018) reported $\mathrm{TiO}_{2}$ NPs with a size between $3.6 \mathrm{~nm}$ and $12 \mathrm{~nm}$ while NPs synthesized by $\mathrm{He}$ et al. (2011) were 10-12 nm in diameter. Jha et al. (2009) reported slightly larger NPs with dimensions ranging from $8 \mathrm{~nm}$ to $20 \mathrm{~nm}$. The synthesis of $\mathrm{TiO}_{2}$ NPs through yeast mediated approaches is mainly based on the biocatalyst molecules from the cell wall. $\mathrm{Ti}^{4+}$ ions are picked up by the electrostatic interaction between them and the negatively charged molecules on the yeast cell surface. $\mathrm{Ti}^{4+}$ interacts with hydroxyl groups on the cell wall and then the yeast/Ti $(\mathrm{OH})_{4}$ structure is converted through calcination into yeast/ $\mathrm{TiO}_{2} \mathrm{NP}$ (He et al., 2011). Also, Jha et al. (2009) proposed that membrane bound oxidoreductases are responsible for the formation of $\mathrm{TiO}_{2} \mathrm{NPs}$ by their strong oxidase activity at low $\mathrm{pH}$ values (Jha et al., 2009). All studies mentioned in this section are summarized in Table 2 together with the characteristics of the obtained NPs and their possible applications.

\section{Plant species involved in the synthesis of $\mathrm{TiO}_{2} \mathrm{NPs}$}

Almost all plants contain a unique and complex combination of chemical compounds which can act as reducing and capping agents, thus being able to mediate the biosynthesis of NPs.
Plant extracts have been used to initiate and support the processes involved in NPs synthesis. This was possible due to the presence of a mixture of organic acids, amino acids, proteins, enzymes, vitamins, polysaccharides and other phytochemicals provided by the plant extract (Rajeshkumar and Bharath, 2017).

There are also other advantages that determined the most important and widespread use of plants in NPs biosynthesis research. So, plant biomass is available almost everywhere on the planet and its diversity is remarkably high. Plant extracts are safe to handle and their use in the synthesis of NPs turns this process into a greener and cost effective one. However, standardizing and implementing the green synthesis of NPs at an industrial scale may face difficulties. There are great differences between plants regarding the nature of their phytochemical content and there is an annual variation of the natural compounds in any plant. Also, $\mathrm{pH}$, temperature, contact time, plant extract concentration and metal precursor salt concentration have to be considered. Still insufficient knowledge regarding how these parameters influence the yield of the synthesis process and the properties of the obtained NPs is available.

In the case of $\mathrm{TiO}_{2}$ NPs synthesis, leaves were the preferred source of phytochemicals probably because they contain the greatest variety of biomolecules with reducing potential. However, some scientists reported the production of $\mathrm{TiO}_{2}$ NPs using roots (Suman et al., 2015; Bavanilatha et al., 2019), fruits (Hossain et al., 2019), seeds (Kashale et al., 2016; Maurya et al., 2019) and parts of plants that are considered agricultural waste (Amanulla and Sundaram, 2019).

When it comes to the extraction, a great number of experiments were performed using water as solvent and only a few chose ethanol (Sundrarajan et al., 2017; Ahmad et al., 2020). $\mathrm{TiO}_{2}$ NPs were synthesised starting from bulk $\mathrm{TiO}_{2}$ (Velayutham et al., 2012; Thakur et al., 2019), but also from different metal salts: $\mathrm{TiO}(\mathrm{OH})_{2}$ (Hudlikar et al., 2012; Zahir et al., 2015), $\mathrm{TiCl}_{4}$ (Kashale et al., 2016; Goutam et al., 2018), titanium tetraisopropoxide (Sankar et al., 2014; Shanavas et al., 2020), titanium oxysulphate (Bavanilatha et al., 2019) or titanium butoxide (Bekele et al., 2020).

Regarding the plant species used, it seems that there is a focus on medicinal plants with healing properties (Ayaz et al., 2017; Unuofin and Lebelo, 2020). NPs can acquire similar properties through the transfer of the phytochemicals on their surface during the capping process. For example, Ahmad et al. (2020) have developed a mint-based method by which spherical NPs have been obtained with a size between 20 and $70 \mathrm{~nm}$, probably due to the presence of alkaloids, terpenoids and phenolics in the Mentha arvensis ethanolic leaf extract (Ahmad et al., 2020). Also Rajkumari et al. (2019) revealed that terpenoids and flavonoids were associated with $\mathrm{TiO}_{2}$ NPs synthesised using Aloe vera leaf extract. Besides medicinal plants, common herbs and spices extracts including Origanum vulgare (Sankar et al., 2014) 
and Cinnamomum tamala (He et al., 2017) were involved in $\mathrm{TiO}_{2}$ NPs biosynthesis.

In general, research has focused on Angiosperm plants while Plantae kingdom comprises also gymnosperms, pteridophytes (ferns), bryophytes (mosses) and macroalgae, and for other kind of NPs these organisms have given efficient results (Acharya and Sarkar, 2014; Bhor et al., 2014; Jha and Prasad, 2010; Momeni and Nabipour, 2015).
To the best of our knowledge, $\mathrm{TiO}_{2}$ NPs were biologically synthesised so far using only bacteria, fungi and angiosperms. $\mathrm{TiO}_{2}$ NPs synthesised through the green approach seem to have a spherical shape in almost every condition. However, their size is fluctuating. In the studies that we took into consideration, the size ranges between $10 \mathrm{~nm}$ (Senthilkumar et al., 2018) and around $200 \mathrm{~nm}$ (Hossain et al., 2019) which is more than the limit of $100 \mathrm{~nm}$ set for describing NPs (Vert et al., 2012).

Table 2. Fungal species involved in $\mathrm{TiO}_{2} \mathrm{NPs}$ synthesis

\begin{tabular}{|c|c|c|c|c|c|}
\hline Fungal species & $\begin{array}{l}\text { Titanium } \\
\text { precursor }\end{array}$ & $\begin{array}{l}\text { Size }(\mathrm{nm}) \\
\text { and shape }\end{array}$ & $\begin{array}{l}\text { Crystalline } \\
\text { phase }\end{array}$ & Application & Reference \\
\hline \multirow[t]{2}{*}{ Aspergillus flavus } & \multirow[t]{2}{*}{ bulk $\mathrm{TiO}_{2}$} & $\begin{array}{l}\text { 12-15; shape } \\
\text { not } \\
\text { mentioned }\end{array}$ & $\begin{array}{c}\text { not } \\
\text { mentioned }\end{array}$ & nano-fertilizer potential & Raliya et al. (2015) \\
\hline & & $\begin{array}{l}62-74 \mathrm{~nm} ; \\
\text { spherical }\end{array}$ & $\begin{array}{l}\text { rutile and } \\
\text { anatase }\end{array}$ & antibacterial activity & Rajakumar et al. (2012a) \\
\hline $\begin{array}{c}\text { Fomes } \\
\text { fomentarius }\end{array}$ & TTIP & $\begin{array}{l}80-120 ; \\
\text { irregular }\end{array}$ & rutile & $\begin{array}{c}\text { antibacterial activity; potential } \\
\text { cytotoxicity against cancer } \\
\text { cells } \\
\end{array}$ & Rehman et al. (2020) \\
\hline \multirow{3}{*}{$\begin{array}{l}\text { Saccharomyces } \\
\text { cerevisiae }\end{array}$} & $\mathrm{TiCl}_{3}$ & $\begin{array}{c}3.6-12 ; \\
\text { spherical }\end{array}$ & anatase & $\begin{array}{l}\text { antibacterial and antifungal } \\
\text { activity } \\
\end{array}$ & Peiris et al. (2018) \\
\hline & $\mathrm{TiCl}_{3}$ & 10-12; oval & anatase & $\begin{array}{l}\text { photodegradation of pollutants } \\
\text { from wastewater }\end{array}$ & He et al. (2011) \\
\hline & $\mathrm{TiO}(\mathrm{OH})_{2}$ & $\begin{array}{c}8-20 \\
\text { spherical }\end{array}$ & $\begin{array}{c}\text { not } \\
\text { mentioned }\end{array}$ & - & Jha et al. (2009) \\
\hline $\begin{array}{l}\text { Trichoderma } \\
\text { viride }\end{array}$ & $\mathrm{TiO}(\mathrm{OH})_{2}$ & $\begin{array}{l}60-86.67 \\
\text { spherical }\end{array}$ & rutile & nano-pesticide potential & $\begin{array}{lll}\begin{array}{l}\text { Chinnaperumal et } \\
(2018)\end{array} & & \\
\end{array}$ \\
\hline
\end{tabular}

TTIP - titanium isopropoxide; $\mathrm{TiCl}_{3}$ - titanium trichloride; $\mathrm{TiO}(\mathrm{OH})_{2}$ - metatitanic acid.

Maurya et al. (2019) have obtained $\mathrm{TiO}_{2}$ NPs with an average diameter of $13 \mathrm{~nm}$ using an ethanolic extract of Bixa orellana seeds. This extract, containing mainly the apocarotenoids bixin and norbixin, was integrated in a sol-gel approach which starts the $\mathrm{TiO}_{2}$ NPs synthesis from titanium butoxide. Using the biomolecules extracted from the seeds of Cicer arietinum, Kashale et al. (2016) synthesised $\mathrm{TiO}_{2} \mathrm{NPs}$ with a size around $14 \mathrm{~nm}$ as shown by particle size distribution histogram performed by TEM. Also, $\mathrm{TiO}_{2}$ NPs with sizes between 10 and $12 \mathrm{~nm}$ were synthesised using leaf extract of the medicinal plant Diospyros ebenum (Senthilkumar et al., 2018). Furthermore, $\mathrm{TiO}_{2}$ NPs of 10-20 nm were prepared using Jatropha curcas leaves extract and were analyzed by SEM (Goutam et al, 2018). A similar result was noticed by Sundrarajan et al. (2017) that used Morinda citrifolia leaves, while root extract of this plant led to larger NPs (Suman et al., 2015). $\mathrm{TiO}_{2} \mathrm{NPs}$ up to $20 \mathrm{~nm}$ were obtained also by using Syzygium cumini leaves extract (Sethy et al., 2020).

Larger particles and more variable in shape were produced by using lemon (Citrus limon) extract, SEM analyses revealing $\mathrm{TiO}_{2}$ structures of 20-200 $\mathrm{nm}$ (Hossain et al., 2019). A large variation was also observed when using the root extract of Glycyrrhiza glabra, the calculated particle size from X-ray diffractogram being $69 \mathrm{~nm}$, but SEM revealed structures with a diameter of
60-140 nm (Bavanilatha et al., 2019). $\mathrm{TiO}_{2} \mathrm{NPs}$ of 25 to $110 \mathrm{~nm}$ were observed using Catharanthus roseus leaf extract (Velayutham et al., 2012). Also, $\mathrm{TiO}_{2} \mathrm{NPs}$, that almost reached the limit of $100 \mathrm{~nm}$ were synthesised using extracts of Artemisia haussknechtii (Alavi and Karimi, 2018), Euphorbia prostrata (Zahir et al., 2015), Vitex negundo (Gandhi et al., 2016) or Moringa oleifera (Sivaranjani and Philominathan., 2016) leaves. In a majority of studies, the cause of obtaining large nanoscale structures is represented by the aggregation of $\mathrm{TiO}_{2}$ NPs as revealed by electronic microscopy (Suman et al., 2015; Gandhi et al., 2016; Goutam et al., 2018). This may indicate a poor-efficiency capping process, an inapropriate concentration of phytochemicals or other unfavourable conditions (Romanello and Fidalgo de Cortalezzi, 2013; Bekele et al., 2020). A selection of studies referring to the plant assisted synthesis of $\mathrm{TiO}_{2}$ NPs is presented in Table 3 together with the characteristics of the obtained NPs and their possible applications. 


\section{Biomedical applications of biologically synthesized $\mathrm{TiO}_{2}$ NPs}

\section{Antimicrobial activity}

As many plants contain phytochemicals with bactericidal effect (Marasini et al., 2015; Todorović et al., 2016), the biosynthesized NPs using them could have a more pronounced antibacterial activity than the conventionally produced ones. Human pathogenic bacteria cause serious problems worldwide as infectious diseases have an alarming rate and raise health concerns (Vouga and Greub, 2016). Clinicians used antibiotics for decades in order to keep under control the pathogenic bacteria. However, the large scale and irresponsible use of antibiotics have led to the occurrence of mutant resistant bacteria (Zaman et al., 2017).

Table 3. Plant species involved in $\mathrm{TiO}_{2} \mathrm{NPs}$ synthesis

\begin{tabular}{|c|c|c|c|c|c|}
\hline Plant source & $\begin{array}{l}\text { Titanium } \\
\text { precursor }\end{array}$ & $\begin{array}{l}\text { Size (nm) and } \\
\text { shape }\end{array}$ & $\begin{array}{l}\text { Crystalline } \\
\text { phase }\end{array}$ & Application & Reference \\
\hline $\begin{array}{l}\text { Aloe vera } \\
\text { aqueous leaf extract }\end{array}$ & $\mathrm{TiCl}_{4}$ & 20-50; spherical & $\begin{array}{c}\text { rutile, } \\
\text { anatase and } \\
\text { brookite }\end{array}$ & antibacterial activity & Rajkumari et al. (2019) \\
\hline $\begin{array}{l}\text { Artemisia haussknechtii } \\
\text { aqueous leaf extract }\end{array}$ & $\mathrm{TiO}(\mathrm{OH})_{2}$ & $\begin{array}{c}92.58 \pm 56.98 \\
\text { spherical } \\
\end{array}$ & $\begin{array}{c}\text { rutile and } \\
\text { anatase }\end{array}$ & $\begin{array}{c}\text { antibacterial and } \\
\text { antioxidant activity }\end{array}$ & Alavi and Karimi (2018) \\
\hline \multirow{2}{*}{$\begin{array}{l}\text { Azadirachta indica } \\
\text { aqueous leaf extract }\end{array}$} & bulk $\mathrm{TiO}_{2}$ & 15-87; spherical & $\begin{array}{c}\text { rutile and } \\
\text { anatase }\end{array}$ & antibacterial activity & Thakur et al. (2019) \\
\hline & TTIP & 124; spherical & amorphous & $\begin{array}{c}\text { photocatalytic } \\
\text { degradation of methyl } \\
\text { red dye }\end{array}$ & Sankar et al. (2015) \\
\hline $\begin{array}{l}\text { Bixa orellana } \\
\text { ethanolic seed extract }\end{array}$ & $\mathrm{Ti}(\mathrm{OBu})_{4}$ & $13 \pm 2 ;$ spherical & anatase & photovoltaic potential & Maurya et al. (2019) \\
\hline $\begin{array}{l}\text { Catharanthus roseus } \\
\text { aqueous leaf extract }\end{array}$ & bulk $\mathrm{TiO}_{2}$ & $\begin{array}{l}\text { 25-110; } \\
\text { irregular }\end{array}$ & $\begin{array}{c}\text { rutile and } \\
\text { anatase }\end{array}$ & $\begin{array}{l}\text { effectiveness against } \\
\text { animal parasites }\end{array}$ & Velayutham et al. (2012) \\
\hline $\begin{array}{l}\text { Cicer arietinum } \\
\text { aqueous seed extract }\end{array}$ & $\mathrm{TiCl}_{4}$ & $\begin{array}{l}\sim 14 \mathrm{~nm} ; \\
\text { spherical }\end{array}$ & $\begin{array}{l}\text { rutile and } \\
\text { anatase }\end{array}$ & $\begin{array}{c}\text { electrochemical } \\
\text { performance in a li-ion } \\
\text { battery }\end{array}$ & Kashale et al. (2016) \\
\hline $\begin{array}{l}\text { Cinnamomum tamala } \\
\text { aqueous leaf extract }\end{array}$ & bulk $\mathrm{TiO}_{2}$ & 23; irregular & anatase & $\begin{array}{l}\text { potential cytotoxicity } \\
\text { against cancer cells }\end{array}$ & He et al. (2017) \\
\hline $\begin{array}{l}\text { Citrus limon } \\
\text { aqueous fruit extract }\end{array}$ & bulk $\mathrm{TiO}_{2}$ & $\begin{array}{l}20-200 ; \\
\text { spherical, } \\
\text { irregular }\end{array}$ & $\begin{array}{l}\text { rutile and } \\
\text { anatase }\end{array}$ & nano-pesticide potential & Hossain et al. (2019) \\
\hline $\begin{array}{l}\text { Diospyros ebenum } \\
\text { aqueous leaf extract }\end{array}$ & TTIP & 10-12; spherical & anatase & $\begin{array}{c}\text { antibacterial activity; } \\
\text { photocatalytic } \\
\text { degradation of crystal } \\
\text { violet dye }\end{array}$ & $\begin{array}{l}\text { Senthilkumar et al. } \\
\text { (2018) }\end{array}$ \\
\hline $\begin{array}{l}\text { Eclipta prostrata } \\
\text { aqueous leaf extract }\end{array}$ & $\mathrm{TiO}(\mathrm{OH})_{2}$ & 36-68; spherical & rutile & - & Rajakumar et al. (2012b) \\
\hline $\begin{array}{l}\text { Euphorbia prostrata } \\
\text { aqueous leaf extract }\end{array}$ & $\mathrm{TiO}(\mathrm{OH})_{2}$ & $\begin{array}{l}83.22 \pm 1.50 \\
\text { spherical }\end{array}$ & anatase & antiparasitic activity & Zahir et al. (2015) \\
\hline $\begin{array}{l}\text { Glycyrrhiza glabra } \\
\text { aqueous root extract }\end{array}$ & $\mathrm{TiOSO}_{4}$ & $\begin{array}{l}60-140 ; \\
\text { spherical }\end{array}$ & anatase & $\begin{array}{l}\text { antibacterial activity; } \\
\text { cytotoxicity assessment }\end{array}$ & Bavanilatha et al. (2019) \\
\hline $\begin{array}{l}\text { Jatropha curcas } \\
\text { aqueous latex extract }\end{array}$ & $\mathrm{TiO}(\mathrm{OH})_{2}$ & $\begin{array}{l}25-50 ; \\
\text { spherical, } \\
\text { irregular }\end{array}$ & anatase & - & Hudlikar et al. (2012) \\
\hline $\begin{array}{l}\text { Jatropha curcas } \\
\text { aqueous leaf extract }\end{array}$ & $\mathrm{TiCl}_{4}$ & 10-20; spherical & anatase & $\begin{array}{c}\text { photocatalytic treatment } \\
\text { of wastewater }\end{array}$ & Goutam et al. (2018) \\
\hline $\begin{array}{l}\text { Kniphofia foliosa } \\
\text { ethanolic root extract }\end{array}$ & $\mathrm{Ti}(\mathrm{OBu})_{4}$ & $\begin{array}{l}\text { not mentioned; } \\
\text { spherical }\end{array}$ & anatase & antibacterial activity & Bekele et al. (2020) \\
\hline $\begin{array}{l}\text { Mangifera indica } \\
\text { aqueous leaf extract }\end{array}$ & $\mathrm{TiO}(\mathrm{OH})_{2}$ & $30 \pm 5 ;$ spherical & rutile & antiparasitic activity & Rajakumar et al. (2015) \\
\hline $\begin{array}{l}\text { Melia azedarach } \\
\text { aqueous leaf extract }\end{array}$ & bulk $\mathrm{TiO}_{2}$ & $\begin{array}{l}\text { 54.12-78.45; } \\
\text { spherical }\end{array}$ & rutile & $\begin{array}{l}\text { nano-fertilizer potential; } \\
\text { photocatalytic } \\
\text { degradation of eosin dye }\end{array}$ & Rafique et al. (2019) \\
\hline $\begin{array}{l}\text { Mentha arvensis } \\
\text { ethanolic leaf extract }\end{array}$ & TTIP & 20-70; spherical & anatase & $\begin{array}{c}\text { antibacterial and } \\
\text { antifungal activity }\end{array}$ & Ahmad et al. (2020) \\
\hline $\begin{array}{l}\text { Mentha aquatica } \\
\text { ethanolic leaf extract }\end{array}$ & TTIP & $\sim 69$; spherical & anatase & $\begin{array}{l}\text { in vitro cytotoxicity and } \\
\text { genotoxicity }\end{array}$ & Koca and Duman (2019) \\
\hline
\end{tabular}


Miu and Dinischiotu / Rev. Biol. Biomed. Sci. 20214 (1) 1-21

\begin{tabular}{|c|c|c|c|c|c|}
\hline $\begin{array}{l}\text { Momordica charantia } \\
\text { aqueous leaf extract }\end{array}$ & $\mathrm{TiCl}_{4}$ & $\sim 70$; irregular & $\begin{array}{c}\text { not } \\
\text { mentioned }\end{array}$ & $\begin{array}{c}\text { potential use in } \\
\text { preventing malaria }\end{array}$ & Gandhi et al. (2018) \\
\hline $\begin{array}{l}\text { Morinda citrifolia } \\
\text { aqueous root extract }\end{array}$ & $\mathrm{TiO}(\mathrm{OH})_{2}$ & $\begin{array}{c}\text { 20.46-39.20; } \\
\text { spherical, oval, } \\
\text { triangular }\end{array}$ & anatase & $\begin{array}{l}\text { effectiveness against } \\
\text { mosquito larvae }\end{array}$ & Suman et al. (2015) \\
\hline $\begin{array}{l}\text { Morinda citrifolia } \\
\text { ethanolic leaf extract }\end{array}$ & $\mathrm{TiCl}_{4}$ & 10-20; spherical & rutile & $\begin{array}{c}\text { antibacterial and } \\
\text { antifungal activity }\end{array}$ & Sundrarajan et al. (2017) \\
\hline $\begin{array}{l}\text { Moringa oleifera } \\
\text { aqueous leaf extract }\end{array}$ & bulk $\mathrm{TiO}_{2}$ & 100; spherical & $\begin{array}{c}\text { not } \\
\text { mentioned }\end{array}$ & wound healing potential & $\begin{array}{l}\text { Sivaranjani and } \\
\text { Philominathan (2016) }\end{array}$ \\
\hline $\begin{array}{l}\text { Origanum vulgare } \\
\text { aqueous leaf extract }\end{array}$ & TTIP & $\begin{array}{l}\text { not mentioned; } \\
\text { spherical }\end{array}$ & amorphous & wound healing potential & Sankar et al. (2014) \\
\hline $\begin{array}{l}\text { Phyllanthus niruri } \\
\text { aqueous leaf extract }\end{array}$ & TTIP & 30-50; spherical & anatase & - & Shanavas et al. (2020) \\
\hline $\begin{array}{l}\text { Prunus yedoensis } \\
\text { aqueous leaf extract }\end{array}$ & bulk $\mathrm{TiO}_{2}$ & 25-50; spherical & $\begin{array}{l}\text { rutile and } \\
\text { anatase }\end{array}$ & $\begin{array}{c}\text { antibacterial activity; } \\
\text { photocatalytic removal } \\
\text { of phosphate }\end{array}$ & Manikandan et al. (2018) \\
\hline $\begin{array}{l}\text { Sesbania grandiflora } \\
\text { aqueous leaf extract }\end{array}$ & bulk $\mathrm{TiO}_{2}$ & $\begin{array}{l}\text { 43-56; } \\
\text { spherical, } \\
\text { square, } \\
\text { triangular }\end{array}$ & $\begin{array}{l}\text { rutile and } \\
\text { anatase }\end{array}$ & toxicity assessment & Srinivasan et al. (2019) \\
\hline $\begin{array}{l}\text { Solanum trilobatum } \\
\text { aqueous leaf extract }\end{array}$ & $\mathrm{TiO}(\mathrm{OH})_{2}$ & 70; spherical & anatase & antiparasitic activity & Rajakumar et al. (2014) \\
\hline $\begin{array}{l}\text { Syzygium cumini } \\
\text { aqueous leaf extract }\end{array}$ & TTIP & 11-18; spherical & anatase & $\begin{array}{l}\text { photocatalytic removal } \\
\text { of lead from wastewater }\end{array}$ & Sethy et al. (2020) \\
\hline $\begin{array}{l}\text { Trigonella foenum- } \\
\text { graecum aqueous leaf } \\
\text { extract }\end{array}$ & $\mathrm{TiOSO}_{4}$ & 20-90; spherical & anatase & $\begin{array}{c}\text { antibacterial and } \\
\text { antifungal activity }\end{array}$ & $\begin{array}{l}\text { Subhapriya and } \\
\text { Gomathipriya (2018) }\end{array}$ \\
\hline $\begin{array}{l}\text { Vitex negundo aqueous } \\
\text { leaf extract }\end{array}$ & $\mathrm{TiCl}_{4}$ & $\begin{array}{c}\sim 93.33 ; \\
\text { spherical }\end{array}$ & rutile & antiparasitic activity & Gandhi et al. (2016) \\
\hline
\end{tabular}

TTIP - titanium isopropoxide; $\mathrm{TiCl}_{4}$ - titanium tetrachloride; $\mathrm{TiO}(\mathrm{OH})_{2}$ - metatitanic acid; $\mathrm{TiOSO}_{4}$ - titanium oxysulfate; $\mathrm{Ti}(\mathrm{OBu})_{4}-$ titanium butoxide.

Microbial resistance became a serious problem nowadays forcing researchers to find new strategies for the control of diseases. NPs may represent a viable alternative to the antibiotics that nowadays cannot be used against multidrug-resistant bacteria. Even though the antimicrobial potential of NPs was first described for those synthesized by physical and chemical methods, biogenic NPs show good antimicrobial activity and depending on their capping biomolecules, their activity may be enhanced. A lot of NPs show antibacterial activity, including $\mathrm{TiO}_{2}$ NPs.

The antimicrobial effect of NPs is of great interest due to its broad-spectrum of action. NPs can be used against pathogenic microorganisms in the field of medicine and/or animal breeding and they can be applied on surfaces, textiles or food packaging (Diaz-Visurraga et al., 2010; Radetić, 2013).

The antibacterial activity of green synthesized $\mathrm{TiO}_{2} \mathrm{NPs}$ was so far assessed on different types of human pathogenic bacteria including the most common Escherichia coli and other gastrointestinal pathogens, but also the ones causing lung infectious diseases (Subhapriya and Gomathipriya, 2018; Bavanilatha et al., 2019; Ahmad et al., 2020; Ağçeli et al., 2020). When it comes to fungal species, the most targeted seems to be Candida albicans (Ağçeli et al., 2020; Peiris et al., 2018; Subhapriya and Gomathipriya, 2018; Sundrarajan et al., 2017), although some experiments tested the antifungal activity of $\mathrm{TiO}_{2}$ NPs against some species of Aspergillus (Ağçeli et al., 2020; Ahmad et al., 2020; Sundrarajan et al., 2017) or Arthrographis cuboid (Ahmad et al., 2020). Using phytochemicals from Kniphofia foliosa plant, Bekele et al. (2020) managed to obtain bio- $\mathrm{TiO}_{2} \mathrm{NPs}$ that were almost as effective as gentamicin, a commonly used antibiotic. The disc diffusion method has revealed that the inhibitory property of these NPs was even higher than gentamicin when applied on S. aureus or Streptococcus pyogenes. $\mathrm{Bio}^{-\mathrm{TiO}_{2}} \mathrm{NPs}$ were not as effective as gentamicin when it comes to E. coli and Klebsiella pneumoniae, but the difference between results was not considerable. This study also showed that an equivalent mixture of the precursor salt and plant extract used for the production of bio- $\mathrm{TiO}_{2} \mathrm{NPs}$ have better results than an unbalanced ratio (Bekele et al. 2020). Similarly, $\mathrm{TiO}_{2}$ NPs synthesized via $A$. hydrophila exhibited a higher inhibitory effect than tetracycline when applied to a series of pulmonary or digestive infectious bacteria (Jayaseelan et al. 2013).

However, a series of studies have reported no or inefficient effect of $\mathrm{TiO}_{2}$ NPs against some of the bacteria mentioned above which means that their antimicrobial activity is highly dependent on the biomolecules provided by the biological entity used. For example, when compared to Ag or $\mathrm{Cu}$ NPs synthesized via $A$. haussknechtii leaf extract, bio- $\mathrm{TiO}_{2} \mathrm{NPs}$ were useless against all bacterial species tested. The study also 
revealed that $\mathrm{TiO}_{2} \mathrm{NPs}$ absorbed on their surface the least amount of phenolics, flavonoids and tannins (Alavi and Karimi, 2018). Furthermore, $\mathrm{TiO}_{2} \mathrm{NPs}$ synthesized using $M$. arvensis had no effect against $E$. coli or $S$. aureus as well as against $A$. cuboid or Aspergillus fumigates fungi (Ahmad et al. 2020). A minor bactericidal effect was reported when $\mathrm{TiO}_{2}$ NPs synthesized using Prunus yedoensis were applied against $S$. aureus and $E$. coli (Manikandan et al. 2018). Depending on the biochemicals used, as well as on the technical parameters (e.g. temperature, $\mathrm{pH}$ ), bio- $\mathrm{TiO}_{2} \mathrm{NPs}$ acquired different antimicrobial properties. This is the reason why an optimization step is desirable when bio- $\mathrm{TiO}_{2} \mathrm{NPs}$ with antimicrobial activity are synthesized.

Regarding to the antifungal activity, biogenic $\mathrm{TiO}_{2} \mathrm{NPs}$ exhibited a moderate effect. As in the case of bacteria, some studies reported no effect (Ağçeli et al. 2020; Ahmad et al. 2020) while others reported almost the same effect as antifungal substance nystatin (Subhapriya and Gomathipriya, 2018). A promising result was achieved with $\mathrm{TiO}_{2}$ NPs synthesized using yeast and applied against Candida albicans. $\mathrm{TiO}_{2}$ NPs exhibited high toxicity in light, but also in dark conditions and this raised their potential to be a future anti-Candida alternative agent. However, the effect against $A$. baumannii was not significantly different compared to the control culture, even though a slight growth inhibition was observed (Peiris et al. 2018).

\section{Antiparasitic activity}

The toxicity of $\mathrm{TiO}_{2}$ NPs may be potentially applied against human and animal parasites. Biogenic $\mathrm{TiO}_{2} \mathrm{NPs}$ were tested in vitro against a series of blood feeding parasites including Pediculus humanus capitis (Rajakumar et al., 2014; Gandhi et al., 2016), Plasmodium falciparum (Gandhi et al., 2018), Leishmania donovani (Zahir et al., 2015), Hippobosca maculate (Velayutham et al., 2012), Bovicola ovis (Velayutham et al., 2012), Hyalomma anatolicum (Rajakumar et al., 2014, 2015), Anopheles subpictus (Rajakumar et al., 2014, 2015; Gandhi et al., 2016), Culex quinquefasciatus (Rajakumar et al., 2015; Suman et al., 2015; Gandhi et al., 2016), Rhipicephalus microplus (Marimuthu et al., 2013; Rajakumar et al., 2015), Haemaphysalis bispinosa (Marimuthu et al., 2013; Rajakumar et al., 2015), Anopheles stephensi (Suman et al., 2015; Gandhi et al., 2018) and Aedes aegypti (Durairaj et al., 2014; Suman et al., 2015). Analyzing all the above cited studies we observed that a concentration between 20 and 25 ppm biogenic- $\mathrm{TiO}_{2} \mathrm{NPs}$ is sufficient to cause a $100 \%$ mortality in 24 hours when applied on the majority of parasites even though the NPs obtained were different in terms of size and crystalline structure. The leaf extract of $C$. roseus was used to produce $\mathrm{TiO}_{2}$ NPs with ability to kill Hippobosca maculate and Bovicola ovis in their adult stage. These parasites are responsible for the spreading of infections to sheep and cattle. The dose that kills $50 \%$ of the parasite population
$\left(\mathrm{LD}_{50}\right)$ has been reported to be around $7 \mathrm{ppm}$. $\mathrm{TiO}_{2} \mathrm{NPs}$ had a significantly improved antiparasitic efficiency compared to the bulk form of $\mathrm{TiO}_{2}$ and the plant extract (Velayutham et al., 2012).

Mosquitoes are a group of insects raising public health concerns worldwide as they are important vectors for many diseases: malaria is spreading through different species of Anopheles including A. subpictus and $A$. stephensi, dengue fever is transmitted by $A$. aegypti and the vector of the West Nile fever is $C$. quinquefasciatus. The mortality of $A$. aegypti second and third instar after exposure to $10 \mathrm{ppm}$ fungal synthesized biogenic- $\mathrm{TiO}_{2}$ NPs was $74 \%$, and $60 \%$ respectively (Durairaj et al., 2014). The $\mathrm{TiO}_{2}$ NPs synthesized by Suman et al. (2015) using $M$. citrifolia root extract were less efficient against A. aegypti. The mortality caused by bio- $\mathrm{TiO}_{2} \mathrm{NPs}$ was related to an increased proteolysis rate and a decline in lipid and carbohydrate content (Durairaj et al., 2014). A. subpictus and $C$. quinquefasciatus fourth instar larvae were killed with $100 \%$ efficiency when 25 ppm $\mathrm{TiO}_{2} \mathrm{NPs}$ obtained with extract of Mangifera indica were applied. The $\mathrm{LD}_{50}$ was around $8 \mathrm{ppm}$ for both species (Rajakumar et al., 2015). These results were also confirmed by Gandhi et al. (2016) with $\mathrm{TiO}_{2} \mathrm{NPs}$ generated by the $V$. negundo extract. $\mathrm{TiO}_{2} \mathrm{NPs}$ synthesized via $S$. trilobatum were more toxic in the case of A. subpictus as $5 \mathrm{ppm}$ killed the entire population, the $\mathrm{LD}_{50}$ being $1.94 \mathrm{ppm}$ (Rajakumar et al., 2014). Contrariwise, $\mathrm{TiO}_{2} \mathrm{NPs}$ synthesized via $M$. citrifolia root were more tolerable for C. quinquefasciatus larvae, the $\mathrm{LD}_{50}$ being around 30 ppm (Suman et al., 2015). The etiological agent of malaria seems to be quite tolerant to $\mathrm{TiO}_{2}$ NPs synthesized using Momordica charantia plant (Gandhi et al., 2018). Chloroquine is a commonly used antimalarial drug which becomes unusable because of a gradual spreading of resistant parasites. Even tested on a chloroquine resistant line of $P$. falciparum, the half maximal inhibitory concentration of the standard drug was around $0.26 \mathrm{ppm}$, while for $\mathrm{TiO}_{2} \mathrm{NPs}$ it was around 60 ppm (Gandhi et al., 2018).

When it comes to the blood feeding acaricides, in most cases, the mortality was also $100 \%$ at a dose of 25 ppm, but $\mathrm{LD}_{50}$ exceeded $10 \mathrm{ppm}$ for the tested species: $R$. microplus, $H$. anatolicum and $H$. bispinosa (Rajakumar et al., 2015). Also, the entire populations of $R$. microplus and $H$. bispinosa larvae and adults could be killed with $16 \mathrm{ppm} \mathrm{TiO}_{2} \mathrm{NPs}$ synthesized using Calotropis gigantea flower extract (Marimuthu et al., 2013). Furthermore, $\mathrm{TiO}_{2}$ NPs synthesized using Solanum trilobatum leaf extract were more toxic for $H$. anatolicum (Rajakumar et al., 2014). The extract of $S$. trilobatum had a more pronounced effect against head louses $(P . h$. capitis) than the extract of $V$. negundo. The proportion was maintained when it comes to the antiparasitic efficiency acquired by NPs synthesized through these extracts. For $S$. trilobatum - $\mathrm{TiO}_{2} \mathrm{NPs}$, the $\mathrm{LD}_{50}$ value was $4.34 \mathrm{ppm}$ while for $V$. negundo $-\mathrm{TiO}_{2} \mathrm{NPs}$, it was $24.32 \mathrm{ppm}$. $\mathrm{TiO}_{2} \mathrm{NPs}$ were more toxic against both the plant extract and NPs 
precursors $\left(\mathrm{TiCl}_{4}\right.$ and bulk $\left.\mathrm{TiO}_{2}\right)($ Rajakumar et al., 2014; Gandhi et al., 2016). The $\mathrm{LD}_{50}$ values for the biogenic$\mathrm{TiO}_{2} \mathrm{NPs}$ mentioned in this section are listed in Table 4.

Table 4. The efficiency of biologically synthesised $\mathrm{TiO}_{2}$ NPs against different parasites

\begin{tabular}{|c|c|c|c|}
\hline Target organism & $\begin{array}{l}\text { Development } \\
\text { stage }\end{array}$ & $\mathbf{L D}_{50}(\mathrm{ppm})$ & Reference \\
\hline \multirow{3}{*}{ Aedes aegypti } & $2^{\text {nd }}$ instar & 6.70 & \multirow{2}{*}{ Durairaj et al. (2014) } \\
\hline & $3^{\text {rd }}$ instar & 8.40 & \\
\hline & larval stage & 23.71 & Suman et al. (2015) \\
\hline \multirow{6}{*}{ Anopheles stephensi } & $1^{\text {st }}$ instar & 2.50 & \multirow{5}{*}{ Gandhi et al. (2018) } \\
\hline & $2^{\text {nd }}$ instar & 2.86 & \\
\hline & $3^{\text {rd }}$ instar & 3.29 & \\
\hline & $4^{\text {th }}$ instar & 3.43 & \\
\hline & Pupa & 5.04 & \\
\hline & larval stage & 13.62 & Suman et al. (2015) \\
\hline \multirow{3}{*}{ Anopheles subpictus } & $4^{\text {th }}$ instage & 7.72 & Rajakumar et al. (2015) \\
\hline & $4^{\text {th }}$ instage & 7.52 & Gandhi et al. (2016) \\
\hline & larval stage & 1.94 & Rajakumar et al. (2014) \\
\hline Bovicola ovis & adult stage & 6.56 & Velayutham et al. (2012) \\
\hline \multirow{3}{*}{ Culex quinquefasciatus } & $4^{\text {th }}$ instar & 8.10 & Rajakumar et al. (2015) \\
\hline & $4^{\text {th }}$ instar & 7.23 & Gandhi et al. (2016) \\
\hline & larval stage & 29.79 & Suman et al. (2015) \\
\hline \multirow{2}{*}{ Haemaphysalis bispinosa } & larval stage & 10.64 & Rajakumar et al. (2015) \\
\hline & adult stage & 9.15 & Marimuthu et al. (2013) \\
\hline Hippobosca maculata & adult stage & 7.09 & Velayutham et al. (2012) \\
\hline \multirow{2}{*}{ Hyalomma anatolicum } & larval stage & 12.11 & Rajakumar et al. (2015) \\
\hline & larval stage & 4.11 & Rajakumar et al. (2014) \\
\hline \multirow{2}{*}{ Pediculus humanus capitis } & adult stage & 24.32 & Gandhi et al. (2016) \\
\hline & adult stage & 4.34 & Rajakumar et al. (2014) \\
\hline $\begin{array}{l}\text { Plasmodium falciparum } \\
\text { chloroquine-sensitive strain 3D7 }\end{array}$ & - & 53.42 & Gandhi et al. (2018) \\
\hline $\begin{array}{l}\text { Plasmodium falciparum } \\
\text { chloroquine-resistant strain INDO }\end{array}$ & - & 59.71 & Gandhi et al. (2018) \\
\hline \multirow{2}{*}{ Rhipicephalus microplus } & larval stage & 13.21 & Rajakumar et al. (2015) \\
\hline & larval stage & 5.43 & Marimuthu et al. (2013) \\
\hline
\end{tabular}

$\mathrm{LD}_{50}$ - dose that causes the death of $50 \%$ of the group of parasites tested.

\section{Antioxidant activity}

Reactive oxygen species (ROS) are generated during intracellular metabolic reactions in mitochondria, peroxisomes and cytosol. The reduction of molecular oxygen with one electron leads to the occurrence of superoxide radical, a primary ROS. This is generated in several reactions catalyzed either by cyclooxygenase, nicotinamide adenine dinucleotide phosphate oxidase or cytochrome c reductase. Superoxide radical is a byproduct of the mitochondrial electron transport chain. Further processes including Fenton reaction or dismutation generate other ROS such as hydroxyl radical or hydrogen peroxide (Manke et al., 2013). Even though ROS generation is a normal process, it can be stimulated by external agents such as pollutants (Lakey et al., 2016) or nanomaterials (Fu et al., 2014). Oxidants are cytotoxic, so homeostasis needs to be maintained by an antioxidant defense system. ROS are neutralized by the action of antioxidant enzymes such as catalase, superoxide dismutase, glutathione peroxidase and reductase as well as by non-enzymatic antioxidants including glutathione, thioredoxin, ascorbate and different vitamins. When antioxidants no longer cope with an abundance of ROS, oxidative stress occurs. The increase of ROS level is

linked with many diseases affecting the nervous, cardiovascular, respiratory, urinary or reproductive systems (Rahman et al., 2012).

Even though there is evidence that standard $\mathrm{TiO}_{2} \mathrm{NPs}$ can produce oxidative stress (Park et al., 2008; Hu et al., 2019), biogenic synthesized $\mathrm{TiO}_{2}$ NPs may react differently due to their corona of phytochemicals and biomolecules. The in vitro approach performed by Alavi and Karimi (2018) showed that biogenic $\mathrm{TiO}_{2}$ NPs exhibit a relatively equal antioxidant activity as the leaf extract of A. haussknechtii that was used in their synthesis. So, $\mathrm{TiO}_{2} \mathrm{NPs}$ would be a good form of packaging the antioxidant biomolecules of the leaf extract. However, this preliminary work showed that green silver and copper NPs have better results when it 
comes to their antioxidant activity (Alavi and Karimi, 2018).

\section{Anticancer effect}

The anticancer activity of $\mathrm{TiO}_{2}$ NPs refers to their toxicity against tumoral cells and so far, it was tested through in vitro approaches. The effects of conventional $\mathrm{TiO}_{2}$ NPs on cancer cells include morphological changes, inhibition of cell proliferation and apoptosis. Proliferation of A549 lung cancer cells was inhibited in a time and dose dependent manner following treatments with $\mathrm{TiO}_{2}$ NPs. The treatment with $200 \mu \mathrm{g} \mathrm{TiO}_{2} \mathrm{NPs} \mathrm{ml}^{-1}$ leads to an inhibitory rate of almost $50 \%$ while apoptosis rate was $20 \%$ (Wang et al., 2015). Most of the studies described a photokilling effect meaning that exposing $\mathrm{TiO}_{2} \mathrm{NPs}$ at UV light for a short period of time enhance their cytotoxicity. Viability of MDA-MB-468 breast cancer cells was reduced by $70 \%$ when treatment with $\mathrm{TiO}_{2} \mathrm{NPs}$ was accompanied by UV irradiation. $\mathrm{TiO}_{2} \mathrm{NPs}$ without UV exposure affected just $45 \%$ of the cell population. The same effect was observed on another breast cancer cell line, MCF-7 (Lagopati et al., 2014). However, $\mathrm{TiO}_{2}$ NPs had no effect on the viability of Caco-2 colon carcinoma cells. Even though intracellular ROS amount was increased after 6 hours, it decreased to an insignificant level after 24 hours (De Angelis et al., 2012).

In general, bio- $\mathrm{TiO}_{2}$ NPs exhibited high cytotoxic activity against cancer cells. Rehman et al. (2020) observed that Fomes fomentarius based $\mathrm{TiO}_{2} \mathrm{NPs}$ caused a massive decrease in the viability of HCT-116 human colorectal carcinoma cells. Up to $90 \%$ of the cells died when they were exposed at $0.5 \mu \mathrm{g}$ bio- $\mathrm{TiO}_{2} \mathrm{ml}^{-1}$ for 48 hours (Rehman et al., 2020). A similar result was also reported by $\mathrm{He}$ et al. (2017) when $C$. tamala extractbased $\mathrm{TiO}_{2}$ NPs decreased the viability of prostatic cancer cells DU145 with more than $60 \%$ after 24 hours (He et al., 2017). Despite these promising results, concerns regarding the non-specificity of $\mathrm{TiO}_{2} \mathrm{NPs}$ could be developed. However, one study gives evidence that tumoral cells may be more sensitive at $\mathrm{TiO}_{2} \mathrm{NPs}$ than normal cells. The IC50 registered by G. glabra $\mathrm{TiO}_{2} \mathrm{NPs}$ was 4 times lower for HepG2 hepatocellular cancer cells than in the case of Vero kidney normal cells (Bavanilatha et al., 2019).

\section{Wound healing potential}

The wound healing potential of bio- $\mathrm{TiO}_{2} \mathrm{NPs}$ may be equally applied on animals and humans. $\mathrm{TiO}_{2}$ is already recognized as a common scaffold used in tissue engineering due to its biocompatibility (Ghosal et al., 2019). In vivo studies on rats suggest that applying $\mathrm{TiO}_{2}$ NPs on skin wounds would accelerate the healing process. $\mathrm{TiO}_{2}$ NPs synthesized using leaf extract from a common spice, $O$. vulgare, were applied daily on a skin excision created at Wistar rats. Results showed that NPs slightly speeded up the wound closure and no bleeding, pus or infection occurred. The pro healing efficacy of bio- $\mathrm{TiO}_{2} \mathrm{NPs}$ was confirmed at tissular and molecular levels. On the $12^{\text {th }}$ day of experiment, the treated wound was characterized by an epithelium with a higher amount of collagen and an increased number of fibroblasts and aggregated macrophages (Sankar et al., 2014).

There are more strategies to apply NPs on skin wounds. The healing process took four days less when the treatment consisted in a collagen film incorporating $\mathrm{TiO}_{2}$ NPs synthesized in a culture of $P$. jensenii (Babitha and Korrapati, 2013). Also, a significant healing effect was revealed when $\mathrm{TiO}_{2}$ NPs were synthesized using $M$. oleifera and applied as a gel-based ointment. The wound healing activity was increased compared to the control, but also to the treatment with sulfadiazine, a standard antibiotic which prevents the occurrence of infections mainly in patients with skin burns (Sivaranjani and Philominathan, 2016).

\section{Agricultural applications of biologically synthesized $\mathrm{TiO}_{2}$ NPs}

Agriculture is a really crucial sector for the existence of our complex socio-economic system. First of all, agricultural practices provide the basic source of food, having a direct impact on national incomes (Praburaj, 2018). Public health is also affected by the nutrient content of different products consumed by humans (Poti et al., 2017). The increase of population we face nowadays results in a continuous increase of food demand, thus a serious pressure on the agro-industrial sector occurs. Moreover, farmers have to deal with climate change and the costs of the high amount of water and energy required for crops. In the past decades, crop yield was increased and maintained high due to a massive use of chemical fertilizers and pesticides. However, these substances have a major disadvantage: they are toxic and can easily accumulate and spread through water, causing issues related to human and environmental health. Furthermore, pest insects or pathogens can evolve resistance strategies against chemical pesticides (Hawkins et al., 2019). Therefore, scientists were forced to find alternatives that may decrease the environmental impact of agricultural practices, but maintain a higher crop production, overall making agriculture more sustainable. Several studies have tried so far to involve $\mathrm{TiO}_{2}$ NPs in crop protection or plant fertilization, the results being promising. Moreover, performing green synthesis methods to obtain NPs for agriculture would lower the environmental impact of this economical sector. Below we described some latest results suggesting that the research and development of nano-fertilizers and nano-pesticides should not be neglected in the future. We identified just a small number of studies regarding the use of biogenic $\mathrm{TiO}_{2}$ NPs in agriculture. 


\section{Nano-pesticides based on biologically synthesized $\mathrm{TiO}_{2} \mathrm{NPS}$}

Nano-pesticides may be useful against two types of target organisms: microorganisms causing diseases and insects that lead to important economic losses. We already discussed the antibacterial and antifungal activity of biogenic $\mathrm{TiO}_{2} \mathrm{NPs}$ against different human pathogens.

Plants can be affected by some strains of the human pathogenic microorganisms that proved to be sensible at $\mathrm{TiO}_{2}$ NPs (Abdulrahman et al., 2020; Schroth et al., 2018). These include strains of Pseudomonas aeruginosa (Jayaseelan et al., 2013; Peiris et al., 2018; Rajkumari et al., 2019; Subhapriya and Gomathipriya, 2018; Sundrarajan et al., 2017) and different strains of Aspergillus (Ağçeli et al., 2020; Ahmad et al., 2020; Sundrarajan et al., 2017) which are also common food contaminants. However, the abovementioned studies were more focused on the medicinal applications of bio$\mathrm{TiO}_{2}$ NPs.

The study of Hossain et al. (2019) is focused on the effect of bio- $\mathrm{TiO}_{2}$ NPs against Dickeya dadantii, a broad host range phytopathogenic bacterium. The biogenic NPs were prepared using an aqueous extract of lemon fruit, TEM images revealing that their size was variable in a range between 20 and $200 \mathrm{~nm}$. When applied on the bacterial cultures, analyses showed that NPs inhibited the growth and motility of $D$. dadantii with up to $60 \%$ and the biofilm formation with 64-66\%. Applying bio- $\mathrm{TiO}_{2}$ NPs on the affected sweet potatoes by this pathogen showed that occurrence of the damaged tissues (maceration zones) was $60 \%$ less likely to happen, showing that efficiency obtained through the in vitro approach was maintained also in vivo. Moreover, this study showed that $\mathrm{TiO}_{2}$ is more effective against pathogenic microorganisms in the nano-scale form taking into consideration that bulk $\mathrm{TiO}_{2}$ was not able to inhibit the growth of $D$. dadantii (Hossain et al., 2019).

Research is not so advanced when it comes to the use of bio- $\mathrm{TiO}_{2}$ for controlling insect attacks, but recent studies suggest that they can affect pests during their larval stages. For example, $\mathrm{TiO}_{2}$ NPs synthesized using the fungus Trichoderma viride caused a serious rate of mortality when applied on Helicoverpa armigera in their first, second and third instar stage. More precisely, a concentration of $100 \mathrm{ppm}$ bio- $\mathrm{TiO}_{2}$ NPs exhibited a mortality between $92 \%$ and $100 \%$ while chemically synthesized $\mathrm{TiO}_{2}$ NPs caused the mortality of $74 \%$ for the $1^{\text {st }}$ instar larvae and just $33 \%$ for the $3^{\text {rd }}$ instar larvae. The pupicidal activity of bio- $\mathrm{TiO}_{2} \mathrm{NPs}$ was also proved to be very high (Chinnaperumal et al., 2018). At the same time, some concerns can be related to the toxicity of $\mathrm{TiO}_{2} \mathrm{NPs}$ on non-target organisms such as organic matter decomposers or pollinators. However, the few studies done so far suggest that bio- $\mathrm{TiO}_{2}$ NPs could be harmless to non-targeted insects. Chinnaperumal et al. (2018) showed that exposing Eudrilus eugeniae earthworms to biosynthesized $\mathrm{TiO}_{2} \mathrm{NPs}$ when placed in an artificial soil would cause no toxic effects. In the same conditions, but in the presence of cypermethrin, earthworms experienced a serious decline in weight and vitality (Chinnaperumal et al., 2018).

Microbial insecticide based on the entomopathogen $B$. thuringiensis is also harmless to non-targeted insects, but when applied on the field, sunlight (including UV radiation), rain or unfavorable temperature make its persistence shorter, affecting its efficiency. To improve the effect of this insecticide, Jalali et al. (2020) added bio- $\mathrm{TiO}_{2}$ NPs on a $B$. thuringiensis culture. They observed that spores from the treated culture exhibited a viability of almost $80 \%$ when exposed to UVA radiation, while non-treated spores' viability was $41 \%$. The highest protective activity was obtained in the case of a mix of anatase and rutile biosynthesized $\mathrm{TiO}_{2}$ NPs. After irradiation, spores were applied on the second-instar larvae of the pest insect Ephestia kuehniella and mortality caused by treated entomopathogens was by $20 \%$ higher than in the case of the non-treated $B$. thuringiensis (Jalali et al., 2020).

Even though there are some studies with interesting and promising results regarding the use of biosynthesized $\mathrm{TiO}_{2}$ NPs in controlling pests, it seems that research has not yet turned its attention to this topic. More studies are needed in order to accurately prove the efficiency of bio$\mathrm{TiO}_{2}$ NPs in protecting crops as well as to assess their toxicity when it comes to the non-targeted organisms including the human population.

\section{Nano-fertilizers based on biologically synthesized $\mathrm{TiO}_{2} \mathrm{NPS}$}

As in the case of chemical fertilizers, the ones based on nanoscale particles can reach the plant's vascular system following their application on the soil or foliar surface. The efficiency of nano-fertilizers is determined by intrinsic factors, including particle size and the particularities of surface coating. There are also extrinsic factors that influence the uptake effectiveness of the nutrients packed as NPs. Soil properties (e.g. texture, $\mathrm{pH}$ ) represent the most important extrinsic factor to be considered when designing new fertilizers. Plant metabolic processes maintaining the organism's vitality, growth rate and production yield strongly depend on a variety of macronutrients (e.g. nitrogen, phosphorus, magnesium, potassium) and micronutrients (e.g. zinc, boron, iron, manganese, copper) (Zulfiqar et al., 2019).

Despite the fact that $\mathrm{TiO}_{2}$ is not a nutrient, the few studies done so far showed that its application on plants could enhance their growth. There is a lack of knowledge regarding the mechanism by which bio- $\mathrm{TiO}_{2}$ NPs intervene in plant development processes. The effect of $\mathrm{TiO}_{2}$ nano-fertilizers on seed germination and plant growth has been assessed mainly in laboratory conditions. Raliya et al. (2015) revealed that, $\mathrm{TiO}_{2} \mathrm{NPs}$ synthesized by extracellular enzymes released by $A$. flavus applied on the foliar surface of Vigna radiata plants lead to the enhancement of shoot and root length 
compared to control ones but this was statistically insignificant.

Another study used $\mathrm{TiO}_{2}$ NPs biosynthesized via Melia azedarach leaf extract applied on the seeds of Pennisetum glaucum. The results showed that bio- $\mathrm{TiO}_{2}$ NPs enhanced the parameters related to seed germination and their efficiency is better than the bulk form of $\mathrm{TiO}_{2}$ (Rafique et al., 2019).

To the best of our knowledge, these are the only two studies that referred to the possible application of bio$\mathrm{TiO}_{2}$ NPs as a fertilizer. Even though the results were not certain and statistically significant, we consider that further studies are needed in order to confirm that $\mathrm{TiO}_{2}$ NPs exhibit no effect on the seed germination and growth performance of economically important plant species.

The use of biologically synthesized $\mathrm{TiO}_{2}$ NPs in environmental management and industrial applications

\section{Photocatalytic removal of pollutants}

The high reactivity and surface area of biogenic NPs make them to act like adsorbents, oxidants, reductants or catalysts. Due to this, their potential to be used in bioremediation processes is important. So far, different biogenic NPs have been involved in the removal of heavy metals (Ehrampoush et al., 2015; Arsiya et al., 2017; Vélez et al., 2018), as well as other organic and inorganic pollutants with a specific focus on toxic dyes (Baruah et al., 2018; David and Moldovan, 2020). NPs may be used in the degradation of toxic chemicals mostly from waters (groundwaters, surface waters or wastewaters), but also from soil (Wang et al., 2019). However, most of the studies are still performed in laboratory conditions and the reported results are preliminary.

$\mathrm{TiO}_{2}$ NPs possess a high catalytic performance which is induced under bright sunlight or ultraviolet irradiation. Several studies showed that the removal of pollutants using bio- $\mathrm{TiO}_{2}$ NPs cannot happen in dark conditions (Manikandan et al., 2018; Senthilkumar et al., 2018; Sethy et al., 2020). So far, the catalytic activity of bio$\mathrm{TiO}_{2}$ NPs was tested against several organic pollutants including methyl red (Sankar et al., 2015), reactive red 31 (Khan and Fulekar, 2016), crystal violet (Senthilkumar et al., 2018) and eosin (Rafique et al., 2019). $\mathrm{TiO}_{2} \mathrm{NPs}$ synthesized using $A$. indica plant proved to have a high photocatalytic activity and caused the degradation of a high amount of methyl red dye in the first hour of treatment (Sankar et al., 2015). In the case of crystal violet dye, the removal was even faster in the presence of $\mathrm{TiO}_{2}$ NPs synthesized using D. ebenum leaves extract (Senthilkumar et al., 2018).

Bacterial synthesized $\mathrm{TiO}_{2}$ NPs seem to require more time for removal of another dye, reactive red 31, but at the end of the 7 hour experiment almost all dye amount was removed (Khan and Fulekar, 2016).
An important result was achieved by Goutam et al. (2018) which designed an experimental system that uses the photocatalytic power of $\mathrm{TiO}_{2} \mathrm{NPs}$ and sunlight in order to be involved in the treatment of tannery wastewater. $\mathrm{TiO}_{2} \mathrm{NPs}$ were synthesized using the leaf extract of $J$. curcas. Previous studies showed that even after a conventional secondary treatment, the wastewater released from tannery facilities exceeded the recommended limit of pollutants when referring to industrial discharge. The secondary treated wastewater that passed through their experimental reactor with these NPs was cleaner and analyses on the chemical oxygen demand revealed that $\mathrm{TiO}_{2} \mathrm{NPs}$ removed pollutants with an efficiency around $82 \%$. Also, the removal efficiency of chromium was found to be at $76.5 \%$ when the reactor operated for 5 hours (Goutam et al., 2018). Beside chromium, bio- $\mathrm{TiO}_{2}$ NPs proved their efficiency in degrading phosphate (Manikandan et al., 2018) and lead removal (Sethy et al., 2020). The photocatalytic activity of $\mathrm{TiO}_{2} \mathrm{NPs}$ synthesized using $S$. cumini leaves was useful in removing lead from the wastewater discharged by an explosive factory. Lead elimination rate was around $82 \%$, but the amount of time required to achieve this value was considerably high (Sethy et al., 2020). In general, bio- $\mathrm{TiO}_{2} \mathrm{NPs}$ exhibited a high pollutant removal yield. However, we consider that the process is not fast enough to be applied at an industrial level and further improvements are needed.

\section{Energy production and storage}

The photocatalytic activity of $\mathrm{TiO}_{2}$ NPs make them suitable for energetical purposes and they could help improving the efficiency of batteries or solar cells. The use of bio- $\mathrm{TiO}_{2}$ NPs in energy storage was explored so far by Kashale et al. (2016). Briefly, their study showed that manufacturing the working electrode of lithium ion batteries from bio- $\mathrm{TiO}_{2}$ NPs would improve the performance of the battery and stability of its storage capacity after a series of recharging cycles (Kashale et al., 2016). Some studies using conventionally synthesized $\mathrm{TiO}_{2}$ NPs have reported similar results (Wu et al., 2014; Wei et al., 2015). When used as a photoanode in a solar cell, bio- $\mathrm{TiO}_{2}$ NPs were not effective in mobilizing electrons. Therefore, the performance of the device was decreased in comparison with the solar cell manufactured using conventionally synthesized $\mathrm{TiO}_{2}$ NPs. However, researchers hypothesized that the coating of calcined biomolecules on the surface of bio- $\mathrm{TiO}_{2} \mathrm{NPs}$ may act as an insulator, constraining the electrical conductivity of the material. So, it was discovered that removing the organic matter from NPs lead to the manufacture of a solar cell with promising performances (ÓrdenesAenishanslins et al., 2014). The transfer of electrons in the solar cell is also facilitated by a pure crystalline phase rather than a mixed phase (Maurya et al., 2019). 


\section{Sustainability of biologically synthesized} $\mathrm{TiO}_{2}$ NPs: environmental, economic and societal implications

The analysis of sustainability is mainly based on the assessment of environmental, economic and societal impacts. A correct evaluation of the sustainability of nanotechnology cannot be done nowadays because of the limited experimental data (Mata et al., 2015). We already presented some advantages which make the biological synthesis of NPs an environmentally friendly method. Referring to the economic impact, we think that involving agrowastes in NPs synthesis would make this process more feasible and cost effective. Some studies already explored this possibility. For example, fruit peels or nutshells from economically important plants are considered food waste materials, although they are a rich source of biomolecules. In an attempt to make agriculture more sustainable and to implement the model of circular economy, research in biotechnology has tried to find a way to use agro-wastes. As in the case of raw plants, agro-waste materials contain active bioproducts that can be easily extracted and used in the synthesis of NPs. Briefly, the extraction protocol consists in grinding, drying and boiling food wastes, thus a low amount of energy is needed.

Normally, all types of NPs can be obtained through biowaste mediated synthesis. So far, wastes involved in NPs production are fruit peels (e.g. banana, orange, lemon, mango, pomegranate, rambutan, groundnut, sugar apple), oak fruit hull, watermelon rind, coconut coir, grape waste, timber industry wastes (e.g. teak leaves) or many others (Adelere and Lateef, 2016).

Table 5. Agro-waste materials involved in $\mathrm{TiO}_{2} \mathrm{NPs}$ synthesis

\begin{tabular}{|c|c|c|c|c|c|}
\hline Agro-waste type & $\begin{array}{l}\text { Titanium } \\
\text { precursor }\end{array}$ & $\begin{array}{l}\text { Size }(\mathrm{nm}) \text { and } \\
\text { shape }\end{array}$ & $\begin{array}{l}\text { Crystalline } \\
\text { phase }\end{array}$ & Application & Reference \\
\hline $\begin{array}{l}\text { Annona squamosa peel } \\
\text { extract }\end{array}$ & $\mathrm{TiO}(\mathrm{OH})_{2}$ & $\begin{array}{l}23 \pm 2 \\
\text { spherical }\end{array}$ & rutile & - & Roopan et al. (2012) \\
\hline Coffee husk & TTIP & $\begin{array}{l}8-10 ; \text { not } \\
\text { mentioned }\end{array}$ & anatase & $\begin{array}{l}\text { remediation of } \\
\text { polluted water }\end{array}$ & Al Qarni et al. (2019) \\
\hline Kiwi peel extract & bulk $\mathrm{TiO}_{2}$ & $\begin{array}{l}54,17-85,13 ; \\
\text { cylindrical }\end{array}$ & anatase & $\begin{array}{c}\text { antibacterial and } \\
\text { antioxidant activity }\end{array}$ & Ajmal et al. (2019) \\
\hline Lemon peel extract & bulk $\mathrm{TiO}_{2}$ & $\begin{array}{l}\text { 80-140; } \\
\text { spherical }\end{array}$ & anatase & $\begin{array}{l}\text { photocatalytic } \\
\text { activity }\end{array}$ & Nabi et al. (2020) \\
\hline Orange peel extract & $\mathrm{TiCl}_{4}$ & $\begin{array}{l}\text { 20-50; not } \\
\text { mentioned }\end{array}$ & anatase & $\begin{array}{c}\text { antibacterial activity } \\
\text { and anticancer } \\
\text { potential }\end{array}$ & $\begin{array}{l}\text { Amanulla and Sundaram } \\
\text { (2019) }\end{array}$ \\
\hline Peach peel extract & bulk $\mathrm{TiO}_{2}$ & $\begin{array}{c}\sim 200 ; \\
\text { cylindrical }\end{array}$ & anatase & $\begin{array}{c}\text { antibacterial and } \\
\text { antioxidant activity }\end{array}$ & Ajmal et al. (2019) \\
\hline Plum peel extract & bulk $\mathrm{TiO}_{2}$ & $\begin{array}{l}47,1-63,21 \\
\text { cylindrical }\end{array}$ & anatase & $\begin{array}{c}\text { antibacterial and } \\
\text { antioxidant activity }\end{array}$ & Ajmal et al. (2019) \\
\hline Rice straw powder & TTIP & $\begin{array}{c}13.0 \pm 3.3 \\
\text { spherical }\end{array}$ & anatase & - & $\begin{array}{l}\text { Ramimoghadam et al. } \\
\text { (2014) }\end{array}$ \\
\hline Tangerine peel extract & TTIP & $\begin{array}{l}50-150 ; \\
\text { spherical }\end{array}$ & $\begin{array}{l}\text { rutile and } \\
\text { anatase }\end{array}$ & - & Rueda et al. (2020) \\
\hline
\end{tabular}

$\mathrm{TiO}(\mathrm{OH})_{2}$ - metatitanic acid; TTIP - titanium isopropoxide; $\mathrm{TiCl}_{4}$ - titanium tetrachloride.

Characteristic for the coffee husk extract is the abundance of caffeine and caffeic acid. Even though FTIR showed that after calcination biochemicals were not absorbed on the surface of NPs, the reduction power is higher than in the case of ethanol as size of NPs show (Al Qarni et al., 2019). Amanulla and Sundaram (2019) have boiled a common organic waste, orange peel, in order to obtain an aqueous extract suitable for the green synthesis of $\mathrm{TiO}_{2} \mathrm{NPs}$. The high concentration orange peel extract was used as a substitute of the sulfuric acid involved in the chemical synthesis method. The authors were able to obtain triangular $\mathrm{TiO}_{2} \mathrm{NPs}$ within a size range of 20 to 50 $\mathrm{nm}$. FTIR analysis has revealed the presence of fatty acids and carbohydrates on the obtained NPs. From the same category of fruits, lemon peel extract showed lower reducing potential as $\mathrm{TiO}_{2} \mathrm{NPs}$ obtained were higher in size (Nabi et al., 2020). Also, NPs synthesized via tangerine peel extract had a variable size range of 50 to $150 \mathrm{~nm}$ (Rueda et al., 2020). Smaller NPs were obtained using Annona squamosa peel extract (Roopan et al., 2012). Using a powder from wasted rice straws also decreased the dimensions of $\mathrm{TiO}_{2}$ NPs. Straws are a rich source of polysaccharides packed in the cellulose structure (Ramimoghadam et al., 2014). Beside agrowastes resulted from plants, there are other biological byproducts that showed potential to be involved in the production of NPs. These include eggshells (Nikhil et al., 2019), honey (Reddy et al., 2012; Haiza et al., 2013) or fish scales (Sinha and Ahmaruzzaman, 2015). Future studies should consider the exploitation of organic waste from households for the manufacture of NPs. A selection of studies referring to the agro-waste assisted synthesis of $\mathrm{TiO}_{2}$ NPs is presented in Table 5 together with the 
characteristics of the obtained NPs and their possible applications.

Regarding the impact on society, the acceptance of NPs by general public and stakeholders is impeded by concerns referring to their safety. The toxicity of green $\mathrm{TiO}_{2}$ NPs against wildlife and human health is not well determined. Srinivasan et al. (2019) have obtained $\mathrm{TiO}_{2}$ NPs through an eco-friendly technique which uses Sesbania grandiflora leaves extract, and they tested their effect on zebrafish embryos. Briefly, $\mathrm{TiO}_{2} \mathrm{NPs}$ affected the eggs surface, and it was reported a delayed hatching and some malformations in the case of a high concentration treatment (Srinivasan et al., 2019). However, experiments on guppy fish showed that $10 \mathrm{mg}$ $\mathrm{I}^{-1} \mathrm{TiO}_{2}$ NPs would not cause any toxic effects (Gandhi et al., 2018). We consider that further studies are needed to evaluate the toxicity of biologically synthesized $\mathrm{TiO}_{2}$ NPs against aquatic and terrestrial ecosystems as well as to identify their possible ways of spreading into natural habitats.

\section{Conclusions}

$\mathrm{TiO}_{2}$ NPs are of great interest due to their numerous and diverse potential applications. However, their most common synthesis methods are associated with environmental issues. Taking into account that it was predicted that production of $\mathrm{TiO}_{2} \mathrm{NPs}$ will increase in the future, scientists have tried to find sustainable ways for their synthesis. Bio-mediated synthesis of $\mathrm{TiO}_{2} \mathrm{NPs}$ was done successfully in a great number of studies and it was proved to be an eco-friendly and cost-effective approach. However, this method remained at a laboratory scale. So far, several studies encouraged the use of green $\mathrm{TiO}_{2} \mathrm{NPs}$ for biomedicinal, agricultural or industrial purposes. The

\section{References}

Abdulrahman I., Aliyu A., Garba L. 2020. Assessment of cereals for fungal contaminants and presence of aflatoxins from two major markets in Kaduna Metropolis. J. Biochem. Microbiol. Biotechnol. 8, 1922.

Acharya K., Sarkar J. 2014. Bryo-synthesis of gold nanoparticles. Int. J. Pharm. Sci. Rev. Res. 29, 82-86.

Adelere I.A., Lateef A., 2016. A novel approach to the green synthesis of metallic nanoparticles: the use of agro-wastes, enzymes, and pigments. Nanotechnol. Rev. 5, 567-587.

Ağçeli G.K., Hammachi H., Kodal S.P., Cihangir N., Aksu Z. 2020. A novel approach to synthesize $\mathrm{TiO}_{2}$ nanoparticles: Biosynthesis by using Streptomyces $s p$. HC1. J. Inorg. Organomet. Polym. Mater. 30, 32213229.

Ahmad R., Mohsin M., Ahmad T., Sardar M. 2015. Alpha amylase assisted synthesis of $\mathrm{TiO}_{2}$ nanoparticles: Structural characterization and antimicrobial, antiparasitic, antioxidant, toxicity against tumoral cells and healing potential of bio- $\mathrm{TiO}_{2} \mathrm{NPs}$ were explored with promising results. Green NPs proved that they still possess photocatalytic properties like chemical ones and so reported good results in experimental devices designed to store and convert solar energy, as well as in treatment of wastewater to get rid of pollutants. Regarding agricultural applications, $\mathrm{TiO}_{2}$ may be effective in controlling pests and for animal healing, but the fertilizer potential was not certainly confirmed so far. These biological activities are mainly determined by the size, shape and cristaline structure of $\mathrm{TiO}_{2} \mathrm{NPs}$. The size of the biogenic $\mathrm{TiO}_{2} \mathrm{NPs}$ varies from one approach to another. The spherical form was predominant, and the majority of studies obtained an anatase crystalline structure. $\mathrm{TiO}_{2} \mathrm{NPs}$ were synthesized by microorganisms including bacteria, microfungi and yeast. In addition, most studies used plants to obtain these NPs. Introducing living organisms into nanotechnological approaches may be the way to make NPs more sustainable. Even if nowadays everyone's attention is mainly focused on environmental issues, sustainability relies also on economical and societal impacts. A correct and complete evaluation of the sustainability of green $\mathrm{TiO}_{2} \mathrm{NPs}$ cannot be done at this moment because of the limited experimental data. Future research should focus on the characteristics of green NPs and how they can be controlled and standardized. Also, it is desirable to find if biochemicals on the surface of green NPs exert a role in their biological activity. Not least, the toxicity of bio$\mathrm{TiO}_{2}$ NPs, as well as their possible routes of bioaccumulation and spreading into environment, need to be examined.

application as antibacterial agents. J. Hazard. Mater. 283, 171-177.

Ahmad W., Jaiswal K.K., Soni S. 2020. Green synthesis of titanium dioxide $\left(\mathrm{TiO}_{2}\right)$ nanoparticles by using Mentha arvensis leaves extract and its antimicrobial properties. Inorg. Nano-Met. Chem. 50, 1032-1038.

Ajmal N., Saraswat K., Bakht M.A., Riadi Y., Ahsan M.J., Noushad M. 2019. Cost-effective and ecofriendly synthesis of titanium dioxide $\left(\mathrm{TiO}_{2}\right)$ nanoparticles using fruit's peel agro-waste extracts: characterization, in vitro antibacterial, antioxidant activities. Green Chem. Lett. Rev. 12, 244-254.

Al Qarni F., Alomair N.A., Mohamed H.H. 2019. Environment-friendly nanoporous titanium dioxide with enhanced photocatalytic activity. Catalysts 9, 799. doi:10.3390/cata19100799

Alavi M., Karimi N. 2018. Characterization, antibacterial, total antioxidant, scavenging, reducing power and ion chelating activities of green synthesized silver, copper and titanium dioxide 
nanoparticles using Artemisia haussknechtii leaf extract. Artif. Cells Nanomed. Biotechnol. 46, 20662081.

Alghuthaymi M.A., Almoammar H., Rai M., Said-Galiev E., Abd-Elsalam K.A. 2015. Myconanoparticles: Synthesis and their role in phytopathogens management. Biotechnol. Biotechnol. Equip. 29, 221236.

Amanulla A.M., Sundaram R. 2019. Green synthesis of $\mathrm{TiO}_{2}$ nanoparticles using orange peel extract for antibacterial, cytotoxicity and humidity sensor applications. Mater. Today 8, 323-331.

Anastas P., Eghbali N. 2010. Green chemistry: Principles and practice. Chem. Soc. Rev. 39, 301-312.

Arsiya F., Sayadi M.H., Sobhani S. 2017. Arsenic (III) adsorption using palladium nanoparticles from aqueous solution. J. Water Environ. Nanotechnol. 2, 166-173.

Ayaz M., Sadiq A., Junaid M., Ullah F., Subhan F., Ahmed J. 2017. Neuroprotective and anti-aging potentials of essential oils from aromatic and medicinal plants. Front. Aging Neurosci. 9, 168. doi:10.3389/fnagi.2017.00168

Babitha S., Korrapati P.S. 2013. Biosynthesis of titanium dioxide nanoparticles using a probiotic from coal fly ash effluent. Mater. Res. Bull. 48, 4738-4742.

Bai H.J., Zhang Z.M., Guo Y., Yang G.E. 2009. Biosynthesis of cadmium sulfide nanoparticles by photosynthetic bacteria Rhodopseudomonas palustris. Colloids Surf. B Biointerfaces 70, 142-146.

Bao S.J., Lei C., Xu M.W., Cai C.J., Jia D.Z. 2012. Environment-friendly biomimetic synthesis of $\mathrm{TiO}_{2}$ nanomaterials for photocatalytic application. Nanotechnology 23, 205601. doi:10.1088/09574484/23/20/205601

Baruah D., Goswami M., Yadav R.N.S., Yadav A., Das A.M. 2018. Biogenic synthesis of gold nanoparticles and their application in photocatalytic degradation of toxic dyes. J. Photochem. Photobiol. B Biol. 186, 5158.

Bavanilatha M., Yoshitha L., Nivedhitha S., Sahithya S. 2019. Bioactive studies of $\mathrm{TiO}_{2}$ nanoparticles synthesized using Glycyrrhiza glabra. Biocatal. Agric. Biotechnol. 19, 101131.

Bekele E.T., Gonfa B.A., Zelekew O.A., Belay H.H., Sabir F.K. 2020. Synthesis of titanium oxide nanoparticles using root extract of Kniphofia foliosa as a template, characterization, and its application on drug resistance bacteria. J. Nanomater. 2020, 2817037.

Bhor G., Maskare S., Hinge S., Singh L., Nalwade A. 2014. Synthesis of silver nanoparticles by using leaflet extract of Nephrolepis exaltata L. and evaluation of antibacterial activity against human and plant pathogenic bacteria. Asian J. Pharm. Technol. Innov. 2, 23-31.

Chinnaperumal K., Govindasamy B., Paramasivam D., Dilipkumar A., Dhayalan A., Vadivel A., Sengodan
K., Pachiappan P. 2018. Bio-pesticidal effects of Trichoderma viride formulated titanium dioxide nanoparticle and their physiological and biochemical changes on Helicoverpa armigera (Hub.). Pestic. Biochem. Physiol. 149, 26-36.

David L., Moldovan B. 2020. Green synthesis of biogenic silver nanoparticles for efficient catalytic removal of harmful organic dyes. Nanomaterials 10, 202. doi:10.3390/nano10020202

De Angelis I., Barone F., Zijno A., Bizzarri L., Russo M.T., Pozzi R., Franchini F., Giudetti G., Uboldi C., Ponti J., Rossi F., De Berardis B. 2012. Comparative study of $\mathrm{ZnO}$ and $\mathrm{TiO}_{2}$ nanoparticles: Physicochemical characterization and toxicological effects on human colon carcinoma cells. Nanotoxicology 7, 1361-1372.

Diaz-Visurraga J., Melendrez M.F., Garcia A., Paulraj M., Cardenas G. 2010. Semitransparent chitosan- $\mathrm{TiO}_{2}$ nanotubes composite film for food package applications. J. Appl. Polym. Sci. 116, 3503-3515.

Durairaj B., Xavier T., Muthu S. 2014. Fungal generated titanium dioxide nanoparticles: A potent mosquito (Aedes aegypti) larvicidal agent. Sch. Acad. J. Biosci. 2, 651-658.

Ehrampoush M.H., Miria M., Salmani M.H., Mahvi A.H. 2015. Cadmium removal from aqueous solution by green synthesis iron oxide nanoparticles with tangerine peel extract. J. Environ. Health Sci. Eng. 13, 84. doi:10.1186/s40201-015-0237-4

Fu P.P., Xia Q., Hwang H.M., Ray P.C., Yu H. 2014. Mechanisms of nanotoxicity: Generation of reactive oxygen species. J. Food Drug Anal. 22, 64-75.

Gandhi P.R., Jayaseelan C., Vimalkumar E., Mary R.R. 2016. Larvicidal and pediculicidal activity of synthesized $\mathrm{TiO}_{2}$ nanoparticles using Vitex negundo leaf extract against blood feeding parasites. J. Asia Pac. Entomol. 19, 1089-1094.

Gandhi P.R., Jayaseelan C., Kamaraj C., Rajasree S.R.R., Mary R.R. 2018. In vitro antimalarial activity of synthesized $\mathrm{TiO}_{2}$ nanoparticles using Momordica charantia leaf extract against Plasmodium falciparum. J. Appl. Biomed. 16, 378-386.

Ghosal K., Agatemor C., Špitálsky Z., Thomas S., Kny E. 2019. Electrospinning tissue engineering and wound dressing scaffolds from polymer-titanium dioxide nanocomposites. Chem. Eng. J. 358, 1262-1278.

Goutam S.P., Saxena G., Singh V., Yadav A.K., Bharagava R.N., Thapa K.B. 2018. Green synthesis of $\mathrm{TiO}_{2}$ nanoparticles using leaf extract of Jatropha curcas L. for photocatalytic degradation of tannery wastewater. Chem. Eng. J. 336, 386-396.

Guilger-Casagrande M., Germano-Costa T., PasquotoStigliani T., Fraceto L.F., Lima R. 2019. Biosynthesis of silver nanoparticles employing Trichoderma harzianum with enzymatic stimulation for the control of Sclerotinia sclerotiorum. Sci. Rep. 9, 14351. doi:10.1038/s41598-019-50871-0 
Haiza H., Azizan A., Mohidin A.H., Halin D.S.C. 2013. Green synthesis of silver nanoparticles using local honey. Nano Hybrids 4, 87-98.

Hawkins N.J., Bass C., Dixon A., Neve P. 2019. The evolutionary origins of pesticide resistance. Biol. Rev. 94, 135-155.

He W., Cui J., Yue Y., Zhang X., Xia X., Liu H., Lui S. 2011. High-performance $\mathrm{TiO}_{2}$ from Baker's yeast. $J$. Colloid Interface Sci. 354, 109-115.

He F., Yu W., Fan X., Jin B. 2017. In vitro cytotoxicity of biosynthesized titanium dioxide nanoparticles in human prostate cancer cell lines. Trop. J. Pharm. Res. 16, 2793-2799.

Hossain A., Abdallah Y., Ali M.A., Masum M.M.I., Li B., Sun G., Meng Y., Wang Y., An Q. 2019. Lemonfruit-based green synthesis of zinc oxide nanoparticles and titanium dioxide nanoparticles against soft rot bacterial pathogen Dickeya dadantii. Biomolecules 9, 863. doi:10.3390/biom9120863

Hou J., Wang Y., Liu J., Zhao J., Long S., Hao J. 2019. Enhanced thermal conductivity of copper- doped polyethylene glycol/urchin- like porous titanium dioxide phase change materials for thermal energy storage. Int. J. Energy Res. 2019, 1-11. doi:10.1002/er.5045

Hu H., Fan X., Yin Y., Guo Q., Yang D., Wei X., Zhang B., Liu J., Wu Q., Oh Y., Chen K., Feng Y., Hou L., Li L., Gu N. 2019. Mechanisms of titanium dioxide nanoparticle-induced oxidative stress and modulation of plasma glucose in mice. Environ. Toxicol. 34, $1221-1235$.

Hudlikar M., Joglekar S., Dhaygude M., Kodam K. 2012. Green synthesis of $\mathrm{TiO}_{2}$ nanoparticles by using aqueous extract of Jatropha curcas L. latex. Mater. Lett. 75, 196-199.

Hulkoti N.I., Taranath T.C. 2014. Biosynthesis of nanoparticles using microbes-A review. Colloids Surf. B Biointerfaces 121, 474-483.

Ijaz I., Gilani E., Nazir A., Bukhari A. 2020. Detail review on chemical, physical and green synthesis, classification, characterizations and applications of nanoparticles. Green. Chem. Lett. Rev. 13, 59-81.

Inshakova E., Inshakov O. 2017. World market for nanomaterials: Structure and trends. MATEC Web Conf. 29, 02013. doi:10.1051/matecconf/201712902013

Jain S., Mehata M.S. 2017. Medicinal plant leaf extract and pure flavonoid mediated green synthesis of silver nanoparticles and their enhanced antibacterial property. Sci. Rep. 7, 15867. doi:10.1038/s41598-01715724-8

Jalali E., Maghsoudi S., Noroozian E. 2020. A novel method for biosynthesis of different polymorphs of $\mathrm{TiO}_{2}$ nanoparticles as a protector for Bacillus thuringiensis from Ultra Violet. Sci. Rep. 10, 426. doi:10.1038/s41598-019-57407-6

Jamkhande P.G., Ghule N.W., Bamer A.H., Kalaskar M.G. 2019. Metal nanoparticles synthesis: An overview on methods of preparation, advantages and disadvantages, and applications. J. Drug. Deliv. Sci. Technol. 53, 101174. doi:10.1016/j.jddst.2019.101174 Jayaseelan C., Rahuman A.A., Roopan S.M., Kirthi A.V., Venkatesan J., Kim S.K., Iyappan M., Siva C. 2013. Biological approach to synthesize $\mathrm{TiO}_{2}$ nanoparticles using Aeromonas hydrophila and its antibacterial activity. Spectrochim. Acta A Mol. Biomol. Spectrosc. 107, 82-89.

Jha A.K., Prasad K. 2010. Green synthesis of silver nanoparticles using Cycas leaf. Int. J. Green Nanotechnol. Phys. Chem. 1, 110-117.

Jha A.K., Prasad K., Kulkarni A.R. 2009. Synthesis of $\mathrm{TiO}_{2}$ nanoparticles using microorganisms. Colloids Surf. B Biointerfaces 71, 226-229.

Johnson J.M., Kinsinger N., Sun C., Li D., Kisailus D. 2012. Urease-mediated room-temperature synthesis of nanocrystalline titanium dioxide. J. Am. Chem. Soc. 134, 13974-13977.

Jose J., Giridhar R., Anas A., Loka Bharathi P.A., Nair S. 2011. Heavy metal pollution exerts reduction/adaptation in the diversity and enzyme expression profile of heterotrophic bacteria in Cochin estuary, India. Environ. Pollut. 159, 2775-2780.

Kalimuthu K., Babu R.S., Venkataraman D., Bilal M., Gurunathan S. 2008. Biosynthesis of silver nanocrystals by Bacillus licheniformis. Colloids Surf. B Biointerfaces 65, 150-153.

Kashale A.A., Gattu K.P., Ghule K., Ingole V.H., Dhanayat S., Sharma R., Chang J.Y., Ghule A.V. 2016. Biomediated green synthesis of $\mathrm{TiO}_{2}$ nanoparticles for lithium ion battery application. Compos. B. Eng. 99, 297-304.

Kaur H., Goyal V., Singh J., Kumar S., Rawat M. 2019. Biomolecules encapsulated $\mathrm{TiO}_{2}$ nano-cubes using Tinospora cordifolia for photodegradation of a textile dye. Micro Nano Lett. 14, 1229-1232.

Khan R., Fulekar M.H. 2016. Biosynthesis of titanium dioxide nanoparticles using Bacillus amyloliquefaciens culture and enhancement of its photocatalytic activity for the degradation of a sulfonated textile dye Reactive Red 31. J. Colloid Interface Sci. 475, 184-191.

Kirthi A.V., Rahuman, A.A., Rajakumar G., Marimuthu S., Santhoshkumar T., Jayaseelan C., Elango G., Zahir A.A., Kamaraj C., Bagavan A. 2011. Biosynthesis of titanium dioxide nanoparticles using bacterium Bacillus subtilis. Mater. Lett. 65, 2745-2747.

Koca F.D., Duman F. 2019. Genotoxic and cytotoxic activity of green synthesized $\mathrm{TiO}_{2}$ nanoparticles. Appl. Nanosci. 9, 815-823.

Kumar V., Gupta R., Ram J., Singh P., Kumar V., Sharma S.K., Katiyar R.S., Kumar R. 2019. High energy $120 \mathrm{MeV} \mathrm{Ti}^{9+}$ ion beam induced modifications in optical, structural and surface morphological properties of titanium dioxide thin films. Vacuum 166, 323-334. 
Kumari M., Mishra A., Pandey S., Singh S.P., Chaudhry V., Mudiam M.K.R., Shukla S., Kakkar P., Nautiyal C.S. 2016. Physico-chemical condition optimization during biosynthesis lead to development of improved and catalytically efficient gold nano particles. Sci. Rep. 6, 27575. doi:10.1038/srep27575

Lagopati N., Tsilibary E.P., Falaras P., Papazafiri P., Pavlatou E.A., Kotsopoulou E., Kitsiou P. 2014. Effect of nanostructured $\mathrm{TiO}_{2}$ crystal phase on photoinduced apoptosis of breast cancer epithelial cells. Int. J. Nanomedicine 9, 3219-3230.

Lakey P., Berkemeier T., Tong H., Arangio A.M., Lucas K., Pöschl U., Shiraiwa M. 2016. Chemical exposureresponse relationship between air pollutants and reactive oxygen species in the human respiratory tract. Sci. Rep. 6, 32916. doi:10.1038/srep32916

Landage K.S., Arbade G.K., Khanna P., Bhongale C.J. 2020. Biological approach to synthesize $\mathrm{TiO}_{2}$ nanoparticles using Staphylococcus aureus for antibacterial and anti-biofilm applications. $J$. Microbiol. Exp. 8, 36-43.

Lopes A.R., Madureira D., Diaz A., Santos S., Vila M.C., Nunes O.C. 2020. Characterisation of bacterial communities from an active mining site and assessment of its potential metal solubilising activity. J. Environ. Chem. Eng. 8, 104495.

Luckarift H.R., Dickerson M.B., Sandhage K.H., Spain, J.C. 2006. Rapid, room-temperature synthesis of antibacterial bionanocomposites of lysozyme with amorphous silica or titania. Small 2, 640-643.

Manikandan V., Velmurugan P., Jayanthi P., Park J.H., Chang W.S., Park Y.J., Cho M., Oh B.T. 2018. Biogenic synthesis from Prunus yedoensis leaf extract, characterization, and photocatalytic and antibacterial activity of $\mathrm{TiO}_{2}$ nanoparticles. Res. Chem. Intermed. 44, 2489-2502.

Manke A., Wang L., Rojanasakul Y. 2013. Mechanisms of nanoparticle-induced oxidative stress and toxicity. Biomed Res. Int. 2013, 942916.

Marasini B.P., Baral P., Aryal P., Ghimire K.R., Neupane S., Dahal N., Singh A., Ghimire L., Shrestha K. 2015. Evaluation of antibacterial activity of some traditionally used medicinal plants against human pathogenic bacteria. Biomed Res. Int. 2015, 265425. doi:10.1155/2015/265425

Marimuthu S., Rahuman A.A., Jayaseelan C., Kirthi A.V., Santhoshkumar T., Velayutham K., Bagavan A., Kamaraj C., Elango G., Iyappan M., Siva C., Karthik L., Rao K.V.B. 2013. Acaricidal activity of synthesized titanium dioxide nanoparticles using Calotropis gigantea against Rhipicephalus microplus and Haemaphysalis bispinosa. Asian Pac. J. Trop. Med. 6, 682-688.

Mata T.M., Martins A.A., Costa C.A.V., Sikdar S.K. 2015. Nanotechnology and sustainability - Current status and future challenges. In: Life Cycle Analysis of Nanoparticles: Risk, Assessment, and
Sustainability, Vaseashta A. (Ed.), Lancaster, Pennsylvania, DEStech Publications Inc, 271-306.

Maurya I.C., Singh S., Senapati S., Srivastava P., Bahadur L. 2019. Green synthesis of $\mathrm{TiO}_{2}$ nanoparticles using Bixa orellana seed extract and its application for solar cells. Sol. Energy 194, 952-958.

Momeni S., Nabipour I. 2015. A simple green synthesis of palladium nanoparticles with Sargassum alga and their electrocatalytic activities towards hydrogen peroxide. Appl. Biochem. Biotechnol. 176, 19371949.

Nabi G., Ain Q.U., Tahir M.B., Riaz K.N., Iqbal T., Rafique M., Hussain S., Raza W., Aslam I., Rizwan M. 2020. Green synthesis of $\mathrm{TiO}_{2}$ nanoparticles using lemon peel extract: their optical and photocatalytic properties. Int. J. Environ. Anal. Chem. doi:10.1080/03067319.2020.1722816

Nikhil P., Sudheer P., Joy P.J., Sruthi T., Vangalpati M. 2019. Synthesis and characterization of nanoparticles using egg shell. Mater. Today 26, 3246-3249.

Órdenes-Aenishanslins N.A., Saona L.A., Durán-Toro V.M., Monrás J.P., Bravo D.M., Pérez-Donoso J.M. 2014. Use of titanium dioxide nanoparticles biosynthesized by Bacillus mycoides in quantum dot sensitized solar cells. Microb. Cell Fact. 13, 90. doi:10.1186/s12934-014-0090-7

Park E.J., Yi J., Chung K.H., Ryu D.Y., Choi J., Park K. 2008. Oxidative stress and apoptosis induced by titanium dioxide nanoparticles in cultured BEAS-2B cells. Toxicol. Lett. 180, 222-229.

Peiris M.M.K., Guansekera T.D.C.P., Jayaweera P.M., Fernando S.S.N. 2018. $\mathrm{TiO}_{2}$ nanoparticles from baker's yeast: A potent antimicrobial. J. Microbiol. Biotechnol. 28, 1664-1670.

Poti J.M., Braga B., Qin B. 2017. Ultra-processed food intake and obesity: What really matters for healthProcessing or nutrient content?. Curr. Obes. Rep. 6, 420-431.

Praburaj L. 2018. Role of agriculture in the economic development of a country. Shanlax Int. J. Commer. 6, $1-5$.

Radetić M. 2013. Functionalization of textile materials with $\mathrm{TiO}_{2}$ nanoparticles. J. Photochem. Photobiol. C Photochem. Rev. 16, 62-76.

Rafique M., Jahangir J., Amin B.A.Z., Tahir M.B., Nabi G., Khan M.I., Khalid N.R., Gillani S.S.A., Sadaf I. 2019. Investigation of photocatalytic and seed germination effects of $\mathrm{TiO}_{2}$ nanoparticles synthesized by Melia azedarach L. leaf extract. J. Inorg. Organomet. Polym. Mater. 29, 2133-2144.

Rahman T., Hosen I., Islam M.M.T., Shekhar H.U. 2012. Oxidative stress and human health. Adv. Biosci. Biotechnol. 3, 997-1019.

Rajakumar G., Rahuman A.A., Roopan S.M., Khanna V.G., Elango G., Kamaraj C., Zahir A.A., Velayutham K. 2012a. Fungus-mediated biosynthesis and characterization of $\mathrm{TiO}_{2}$ nanoparticles and their 
activity against pathogenic bacteria. Spectrochim. Acta A Mol. Biomol. Spectrosc. 91, 23-29.

Rajakumar G., Rahuman A.A., Priyamvada B., Khanna V.G., Kumar D.K., Sujin P.J. 2012b. Eclipta prostrata leaf aqueous extract mediated synthesis of titanium dioxide nanoparticles. Mater. Lett. 68, 115-117.

Rajakumar G., Rahuman A.A., Jayaseelan C., Santhoshkumar T., Marimuthu S., Kamaraj C., Bagavan A., Zahir A.A., Kirthi A.V., Elango G., Arora P., Karthikeyan R., Manikandan S., Jose S. 2014. Solanum trilobatum extract-mediated synthesis of titanium dioxide nanoparticles to control Pediculus humanus capitis, Hyalomma anatolicum anatolicum and Anopheles subpictus. Parasitol. Res. 113, 469479.

Rajakumar G., Rahuman A.A., Roopan S.M., Chung I.M., Anbarasan K., Karthikeyan V. 2015. Efficacy of larvicidal activity of green synthesized titanium dioxide nanoparticles using Mangifera indica extract against blood-feeding parasites. Parasitol. Res. 114, 571-581.

Rajeshkumar S., Bharath L.V. 2017. Mechanism of plantmediated synthesis of silver nanoparticles $-\mathrm{A}$ review on biomolecules involved, characterisation and antibacterial activity. Chem. Biol. Interact. 273, 219227.

Rajkumari J., Magdalane C.M., Siddhardha B., Madhavan J., Ramalingam G., Al-Dhabi N.A., Arasu M.V., Ghilan A.K.M., Duraipandiayan V., Kaviyarasu K. 2019. Synthesis of titanium oxide nanoparticles using Aloe barbadensis mill and evaluation of its antibiofilm potential against Pseudomonas aeruginosa PAO1. J. Photochem. Photobiol. B Biol. 201, 111667. doi:10.1016/j.jphotobiol.2019.111667

Raliya R., Biswas P., Tarafdar J.C. 2015. $\mathrm{TiO}_{2}$ nanoparticle biosynthesis and its physiological effect on mung bean (Vigna radiata L.). Biotechnol. Rep. 5 , 22-26.

Ramimoghadam D., Bagheri S., Abd Hamid S.B. 2014. Biotemplated synthesis of anatase titanium dioxide nanoparticles via lignocellulosic waste material. Biomed Res. Int. 2014, 205636.

Rana A., Yadav K., Jagadevan S. 2020. A comprehensive review on green synthesis of nature-inspired metal nanoparticles: Mechanism, application and toxicity. $J$. Clean. Prod. 272, 122880.

Reddy S.M., Datta K.K.R., Sreelakshmi C., Eswaramoorthy M., Reddy B.V.S. 2012. Honey mediated green synthesis of Pd nanoparticles for suzuki coupling and hydrogenation of conjugated olefins. Nanosci. Nanotechnol. Lett. 4, 420-425.

Rehman S., Farooq R., Jermy R., Asiri S.M., Ravinayagam V., Al Jindan R., Alsalem Z., Shah M.A., Reshi Z., Sabit H., Khan F.A. 2020. A wild Fomes fomentarius for biomediation of one pot synthesis of titanium oxide and silver nanoparticles for antibacterial and anticancer application. Biomolecules 10, 622. doi:10.3390/biom 10040622
Robichaud C.O., Uyar A.E., Darby M.R., Zucker L.G., Wiesner M.R. 2009. Estimates of upper bounds and trends in nano- $\mathrm{TiO}_{2}$ production as a basis for exposure assessment. Environ. Sci. Technol. 43, 4227-4233.

Rodríguez-González V., Terashima C., Fujishima A. 2019. Applications of photocatalytic titanium dioxidebased nanomaterials in sustainable agriculture. $J$. Photochem. Photobiol. C Photochem. Rev. 40, 49-67.

Romanello M.B., Fidalgo de Cortalezzi M.M. 2013. An experimental study on the aggregation of $\mathrm{TiO}_{2}$ nanoparticles under environmentally relevant conditions. Water Res. 47, 3887-3898.

Roopan S.M., Bharathi A., Prabhakarn A., Rahuman A.A., Velayutham K., Rajakumar G., Padmaja R.D., Lekshmi M., Madhumitha G. 2012. Efficient phytosynthesis and structural characterization of rutile $\mathrm{TiO}_{2}$ nanoparticles using Annona squamosa peel extract. Spectrochim. Acta A Mol. Biomol. Spectrosc. 98, 8690.

Rueda D., Arias V., Zhang Y., Cabot A., Agudelo A.C., Cadavid D. 2020. Low-cost tangerine peel waste mediated production of titanium dioxide nanocrystals: Synthesis and characterization. Environ. Nanotechnol. Monit. Manag. 13, 100285.

Sankar R., Dhivya R., Shivashangari K.S., Ravikumar V. 2014. Wound healing activity of Origanum vulgare engineered titanium dioxide nanoparticles in Wistar Albino rats. J. Mater. Sci. Mater. Med. 25, 17011708 .

Sankar R., Rizwana K., Shivashangari K.S., Ravikumar V. 2015. Ultra-rapid photocatalytic activity of Azadirachta indica engineered colloidal titanium dioxide nanoparticles. Appl. Nanosci. 5, 731-736.

Satpathy S., Patra A., Ahirwar B., Hussain M.D. 2019. Process optimization for green synthesis of gold nanoparticles mediated by extract of Hygrophila spinosa $\mathrm{T}$. Anders and their biological applications. Physica E Low Dimens. Syst. Nanostruct. 121, 113830. doi:10.1016/j.physe.2019.113830

Schroth M.N., Cho J.J., Green S.K., Kominos S.D. 2018. Epidemiology of Pseudomonas aeruginosa in agricultural areas. J. Med. Microbiol. 67, 1191-1201.

Senthilkumar S., Ashok M., Kashinath L., Sanjeeviraja C., Rajendran A. 2018. Phytosynthesis and characterization of $\mathrm{TiO}_{2}$ nanoparticles using Diospyros ebenum leaf extract and their antibacterial and photocatalytic degradation of crystal violet. Smart Sci. 6, 1-9.

Sethy N.K., Arif Z., Mishra P.K., Kumar P. 2020. Green synthesis of $\mathrm{TiO}_{2}$ nanoparticles from Syzygium cumini extract for photo-catalytic removal of lead $(\mathrm{Pb})$ in explosive industrial wastewater. Green Process. Synth. 9, 171-181.

Shanavas S., Priyadharsan A., Karthikeyan S., Dharmaboopathi K., Ragavan I., Vidya C., Acevedo R., Anbarasana P.M. 2020. Green synthesis of titanium dioxide nanoparticles using Phyllanthus niruri leaf extract and study on its structural, optical 
and morphological properties. Mater. Today 26 , 3531-3534.

Shankar S., Chorachoo J., Jaiswal L., Voravuthikunchai S.P. 2014. Effect of reducing agent concentrations and temperature on characteristics and antimicrobial activity of silver nanoparticles. Mater. Lett. 137, 160163.

Singh A., Vihinen J., Frankberg E., Hyvärinen L., Honkanen M., Levänen E. 2016. Pulsed laser ablation-induced green synthesis of $\mathrm{TiO}_{2}$ nanoparticles and application of novel small angle $\mathrm{X}$ ray scattering technique for nanoparticle size and size distribution analysis. Nanoscale Res. Lett. 11, 447. doi:10.1186/s11671-016-1608-1

Sinha T., Ahmaruzzaman M. 2015. Green synthesis of copper nanoparticles for the efficient removal (degradation) of dye from aqueous phase. Environ. Sci. Pollut. Res. 22, 20092-20100.

Sivaranjani V., Philominathan P. 2016. Synthesize of titanium dioxide nanoparticles using Moringa oleifera leaves and evaluation of wound healing activity. Wound Med. 12, 1-5.

Sofronov D., Rucki M., Demidov O., Doroshenko A., Sofronova E., Shaposhnyk A., Kapustnik O., Mateychenko P., Kucharczyk W. 2020. Formation of $\mathrm{TiO}_{2}$ particles during thermal decomposition of $\mathrm{Ti}\left(\mathrm{NO}_{3}\right)_{4}, \mathrm{TiOF}_{2}$ and $\mathrm{TiOSO}_{4}$. J. Mater. Res. Technol. $9,12201-12212$.

Srinivasan M., Venkatesan M., Arumugam V., Natesan G., Saravanan N., Murugesan S., Ramachandran S., Ayyasamy R., Pugazhendhi A. 2019. Green synthesis and characterization of titanium dioxide nanoparticles $\left(\mathrm{TiO}_{2} \mathrm{NPs}\right)$ using Sesbania grandiflora and evaluation of toxicity in zebrafish embryos. Process Biochem. 80, 197-202.

Subhapriya S., Gomathipriya P. 2018. Green synthesis of titanium dioxide $\left(\mathrm{TiO}_{2}\right)$ nanoparticles by Trigonella foenum-graecum extract and its antimicrobial properties. Microb. Pathog. 116, 215-220.

Suman T.Y., Ravindranath R.R.S., Elumalai D., Kaleena P.K., Ramkumar R., Perumal P., Aranganathan L., Chitrarasu P. S. 2015. Larvicidal activity of titanium dioxide nanoparticles synthesized using Morinda citrifolia root extract against Anopheles stephensi, Aedes aegypti and Culex quinquefasciatus and its other effect on non-target fish. Asian Pac. J. Trop. Dis. 5, 224-230.

Sundrarajan M., Bama K., Bhavani M., Jegatheeswaran S., Ambika S., Sangili A., Nithya P., Sumathi R. 2017. Obtaining titanium dioxide nanoparticles with spherical shape and antimicrobial properties using $M$. citrifolia leaves extract by hydrothermal method. $J$. Photochem. Photobiol. B Biol. 171, 117-124.

Taran M., Rad M., Alavi M. 2018. Biosynthesis of $\mathrm{TiO}_{2}$ and $\mathrm{ZnO}$ nanoparticles by Halomonas elongata IBRCM 10214 in different conditions of medium. Bioimpacts 8, 81-89.
Tehrani A.A., Omranpoor M.M., Vatanara A., Seyedabadi M., Ramezani V. 2019. Formation of nanosuspensions in bottom-up approach: theories and optimization. DARU J. Pharm. Sci. 27, 451-473.

Thakur B.K., Kumar A., Kumar D. 2019. Green synthesis of titanium dioxide nanoparticles using Azadirachta indica leaf extract and evaluation of their antibacterial activity. S. Afr. J. Bot. 124, 223-227.

Todorović B., Potočnik I., Rekanović E., Stepanović M., Kostić M., Ristić M., Milijašević-Marčić S. 2016. Toxicity of twenty-two plant essential oils against pathogenic bacteria of vegetables and mushrooms. $J$. Environ. Sci. Health B 51, 832-839.

Unuofin J.O., Lebelo S.L. 2020. Antioxidant effects and mechanisms of medicinal plants and their bioactive compounds for the prevention and treatment of type 2 diabetes: An updated review. Oxid. Med. Cell. Longev. 2020, 1356893. doi:10.1155/2020/1356893

Velayutham K., Rahuman A.A., Rajakumar G., Santhoshkumar T., Marimuthu S., Jayaseelan C., Bagavan A., Kirthi A.V., Kamaraj C., Zahir A.A., Elango G. 2012. Evaluation of Catharanthus roseus leaf extract-mediated biosynthesis of titanium dioxide nanoparticles against Hippobosca maculata and Bovicola ovis. Parasitol. Res. 111, 2329-2337.

Vélez E., Campillo G., Morales G., Hincapié C., Osorio J., Arnache O. 2018. Silver nanoparticles obtained by aqueous or ethanolic Aloe vera extracts: An assessment of the antibacterial activity and mercury removal capability. J. Nanomater. 2018, 7215210. doi:10.1155/2018/7215210

Velgosová O., Mražíková A., Marcinčáková R. 2016. Influence of $\mathrm{pH}$ on green synthesis of $\mathrm{Ag}$ nanoparticles. Mater. Lett. 180, 336-339.

Vert M., Doi Y., Hellwich K.H., Hess M., Hodge P., Kubisa P., Rinaudo M., Schué F. 2012. Terminology for biorelated polymers and applications (IUPAC Recommendations 2012). Pure Appl. Chem. 84, 377410.

Vouga M., Greub G. 2016. Emerging bacterial pathogens: The past and beyond. Clin. Microbiol. Infect. 22, 12-21.

Wang Y., Cui H., Zhou J., Li F., Wang J., Chen M., Liu Q. 2015. Cytotoxicity, DNA damage, and apoptosis induced by titanium dioxide nanoparticles in human non-small cell lung cancer A549 cells. Environ. Sci. Pollut. Res. 22, 5519-5530.

Wang Y., O'Connor D., Shen Z., Lo I.M.C., Tsang D.C.W., Pehkonen S., Pu S., Hou D. 2019. Green synthesis of nanoparticles for the remediation of contaminated waters and soils: Constituents, synthesizing methods, and influencing factors. $J$. Clean. Prod. 226, 540-549.

Wei J., Liu J.X., Wu Z.Y., Zhan Z.L., Shi J., Xu K. 2015. Research on the electrochemical performance of rutile and anatase composite $\mathrm{TiO}_{2}$ nanotube arrays in lithium-ion batteries. J. Nanosci. Nanotechnol. 15, 5013-5019. 
Wong-Pinto L., Menzies A., Ordóñez J.I. 2020. Bionanomining: biotechnological synthesis of metal nanoparticles from mining waste-opportunity for sustainable management of mining environmental liabilities. Appl. Microbiol. Biotechnol. 104, 18591869.

Wu H.Y., Hon M.H., Kuan C.Y., Leu I.C. 2014. Preparation of $\mathrm{TiO}_{2}(\mathrm{~B})$ nanosheets by a hydrothermal process and their application as an anode for lithiumion batteries. J. Electron. Mater. 43, 1048-1054.

Wu F., Zhou Z., Hicks A.L. 2019. Life cycle impact of titanium dioxide nanoparticle synthesis through physical, chemical, and biological routes. Environ. Sci. Technol. 53, 4078-4087.

Yadav B.C., Singh S., Yadav T.P. 2015. Titania prepared by ball milling: Its characterization and application as liquefied petroleum gas sensor. Synth. React. Inorg. Met.-Org. Nano-Met. Chem. 45, 487-494.

Zahir A.A., Chauhan I.S., Bagavan A., Kamaraj C., Elango G., Shankar J., Arjaria N., Roopan S.M., Rahuman A.A., Singh N. 2015. Green synthesis of silver and titanium dioxide nanoparticles using Euphorbia prostrata extract shows shift from apoptosis to $G_{0} / G_{1}$ arrest followed by necrotic cell death in Leishmania donovani. Antimicrob. Agents Chemother. 59, 4782-4799.

Zaman S.B., Hussain M.A., Nye R., Mehta V., Mamun K.T., Hossain N. 2017. A review on antibiotic resistance: Alarm bells are ringing. Cureus 9, 1403. doi:10.7759/cureus. 1403

Zamani A., Marjani A.P., Zahra M. 2019. Agricultural waste biomass-assisted nanostructures: Synthesis and application. Green Process Synth. 8, 421-429.

Ziental D., Czarczynska-Goslinska B., Mlynarczyk D.T., Glowacka-Sobotta A., Stanisz B., Goslinski T., Sobotta L. 2020. Titanium dioxide nanoparticles: Prospects and applications in medicine. Nanomaterials 10, 387. doi:10.3390/nano10020387

Zulfiqar F., Navarro M., Ashraf M., Akram N.A., Munné-Bosch S. 2019. Nanofertilizer use for sustainable agriculture: Advantages and limitations. Plant Sci. 289, 110270. 